\title{
MONITORING LAND COVER CHANGES OF THE NORTH- WESTERN AREA AT EL FAYOUM DEPRESSION AND ITS SOIL SUITABILITY FOR AGRICULTURAL PURPOSES
}

\author{
Tolba S. Abdel Aal and Abdel Aziz B.A. Belal*
}

Soils and Water Dept., Fac. of Agric., El Fayoum University, Egypt

*National Authority for Remote Sensing and Space Sciences, Egypt

\section{ABSTRACT:}

This study is a trail for monitoring land cover changes by using Landsat Imagery technique as well as determining soil suitability for irrigated agriculture at the north-western outskirt of El-Fayoum depression during a period of 19842005. The studied area lies between latitudes of $29^{\circ} 19^{\prime \prime}$ and $29^{\circ} 26^{\prime \prime} \mathrm{N}$ and longitudes of $30^{\circ} 24^{\prime \prime}$ and $30^{\circ} 34$ "E. To achieve this target, Landsat Imagery technique was carried out using two Landsat Thematic Mapper and Enhance Thematic Mapper scenes acquired on 1984 and 2005 covering the north-western part of El-Fayoum depression have been processed. A supervised Spectral Angler Mapper approach was also applied to classify the images into four land use/cover classes, i.e., urban, agriculture, water and desert areas. It was observed that some desert areas had been transformed into agriculture and settlement during the investigated period of twenty one year (1984-2005).

This study clarified that the reclaimed area for agriculture purposes during the studied period reached 1726.91 ha, which constitute about $8.15 \%$ of the total area under study and nation settlement (arbanization) increased with about 310.06 ha, which couple nearly $1.46 \%$ of the total area. Whereas, the water area was promoted with $31.48 \mathrm{ha}$, which equal about $0.22 \%$. All the land cover units were increased account on the expanse of desert unit, which decreased with 2097.20 ha (9.78\% of the total studied area). These increases are more attributed to the geophysical and anthropogenic processes such as salinity, alkalinity, water logging, wind erosion, reclamation of new agricultural land and urbanization.

The satellite image interpretation map of the year 2005 was identified into two main landscapes and thirteen landform units, based on the visual interpretation of Landsat data ETM7 (Enhanced Thematic Mapper 7) and applying the Landscape feature approaches. The validity of physiographic map bounders were field checked to represent the different soil mapping units with model soil profiles. Thirty-two mini pits were located and studied for setting up the physiographic boundaries and characteristic of soil map legend. Also, the variations of soil characteristics between the main identified physiographic units were represented by eighteen soil profiles, which chosen to be full morphologically described according to USDA (2003).

Soil taxa were surveyed according to the USDA (1975 and 2010), and the studied soils could be categorized into three orders and six sub-groups, as follows: Aridisols (Typic Calcigypsids, Lithic Calcigypsids, and Typic Haplocalcids), Entisols (Typic Torriorthents and Typic Torrifluvents) and Vertisols (Typic Haplotorrierts). According to the parametric system undertaken by Sys and Verheye (1978), soil suitability classes of the studied area could be categorized into four classes, i.e., highly (S1), moderately (S2), marginally (S3) and not suitable (N1 \& N2).

Key words: El-Fayoum soils, land cover changes detection, monitoring land reclamation process and land evaluation.

Fayoum J. Agric. Res. \& Dev., Vol.25, No.1, January, 2011 


\section{INTRODUCTION:}

The rapid population growth in Egypt will probably be the most important reason for the horizontal expansion in agriculture and achieving sustainable agricultural development. The strategy of El Fayoum Governorate for the horizontal expansion in agriculture needs more suitable land resources to meet progressive increase of human pressure on the limited cultivated area. Fortunately the governmental policy has already taken great strikes towards the agricultural expansion; espically in the areas adjacent and surrounding the old cultivated areas and urbanizing the desert. Many private and public sectors investments were paid in reclaiming soils around El Fayoum depression, especially with the facilities of irrigation water. The north-western desert outskirt of the closed internal drainage Fayoum depression represents one of the main promising land resources, due to the availability of supplementary irrigation water which could be partly balanced by the reuse of drainage water of El Rayan drain after mixing with the Nile water from Bahr kasr El Gebaly and Bahr Qaroun canals. Due to the fast expansion in reclamation and the excessive urbanization, El-Fayoum governorate has created a new governmental district (Youssif El Sedeek) to be distinct to the area.

It is well known that the outskirts of El Fayoum Governorate are mainly desert areas and have less productive desert sandy or calcareous soils. Such promising areas of virgin sandy and calcareous soils are commonly known to have a poor soil structure, low water holding capacity and limited fertility. This is due to the fact that their main mechanical constituents are sand fraction and its high content of active $\mathrm{CaCO} 3$ that affect distinctly different soil properties related to plant growth, i.e., crusting, soil-water relations, availability of plant nutrients, etc... (Ragab, 2001). The studied area lies within the arid desert belt and characterized by the continental climate. The climatelogical data indicate that the area under investigation is dry and hot in summer and slightly cold in winter, the principal meteorological data recorded at El-Fayoum Station as the last sixth years average reveal that the mean annual temperature ranges between 12.3 and $33.1^{\circ} \mathrm{C}$, the annual maximum temperature differs from 22.2 to $37.6^{\circ} \mathrm{C}$ and the minimum ranges from 9.2 to $13.7^{\circ} \mathrm{C}$. The studied area receives a very low amount of rainfall, where the average rate ranges from 14 to $21 \mathrm{~mm} /$ year. The mean annual relative humidity ranges from 47 to $60 \%$. Wind velocity ranges from 3.0 to $4.1 \mathrm{~km} / \mathrm{hr}$. The rate of evaporation ranges from $3 \mathrm{~mm} /$ day in winter to $11-13 \mathrm{~mm} /$ day in summer.

Remotely sensed data have been widely used for environment change study in the past decades and large collections of remote sensing imagery have made it possible to analyze long-term change of environmental elements and impact of human activities. Research has been widely reported on methodology of remote sensing change detection and monitoring (i.e., Singh 1989, MacLeod and Congalton 1998, Mas 1999, Liu et al. 2004). The Spectral Angle Mapper (SAM) method allows single-step matching of pixel spectra to reference spectra in ndimensional spectral space (Kruse, 1999). The effects of shadow on the final classification image can essentially be eliminated using this technique. Moreover, this method can also be applied to discrimination of vegetated surfaces which reflect incident solar radiation anisotropically (Yuhas $\boldsymbol{e t}$. $\boldsymbol{a l}$, 1992). On the other hand, the basic idea of the object-based classification is to classify group of pixels that represent real objects. Neighboring pixels are grouped into regions or segments based on similarity criteria - digital number, texture - in a process referred to as segmentation, (Congalton,1991).

Change detection approaches can be characterized into two broad groups, namely, bi-temporal change detection and temporal trajectory analysis (Coppin $\boldsymbol{e t}$

Fayoum J. Agric. Res. \& Dev., Vol.25, No.1, January, 2011 
al. 2004). The former measures land cover changes based on a 'two-epoch' timescale, i.e. the comparison between two dates. Even if land cover information sometimes is acquired for more than two epochs, the changes are still measured on the basis of pairs of dates. The latter analyses the changes based on a 'continuous' timescale, i.e. the focus of the analysis is not only on what has changed between dates, but also on the progress of the change over the period. At present, most change detection methods belong to bi-temporal change detection approach including, for example, image differencing (Weismiller et al. 1977), vegetation index differencing (Liu et. al., 2002), change vector analysis (CVA) (Malila, 1980, Lunetta et al. 2004), principal component analysis (PCA), (Liu et al. 2004), postclassification comparison (Weismiller et al. 1977), multi-temporal composite and classification (Zhao et al. 2003), and artificial neural network (ANN) (Dai and Khorram 1999).

From a scientific point of view, the data of the current work must come from the identified physiographic features of a unique area in Egypt by mapping them to be in a harmony of physiography and soil data set, serving the extrapolation approach when other areas will be under study. It is also to find the best adaptation between certain land units with agricultural utilization projects to give the maximum output. The evaluation of soil potentialities of the identified main physiographic units through determining their main characteristics may be helpful in supporting the local knowledge, especially for identifying the best land use under irrigated agriculture for achieving an agricultural utilization policy of the region under consideration. For this purpose, the harmony of descriptive and processing systems, established by Sys and Verheye (1978) can be used.

The current study aims to monitor desert and cultivated land degradation processes in the north-west outskirt of El Fayoum depression. Satellite visualinterpretation, integrated with geographic information system (GIS) was employed in the current study. A special attention will be paid to the expansion in cultivated areas, urban expansion of the main towns or villages, soil degradation due to salinization and sand encroachment over the arable lands during the period of 1984 -2005. In addition, the present work is undertaken to evaluate the soil potentialities of the identified main physiographic units through determining their main soil characteristics and to update and support the local knowledge, particularly the best use of land whether be under demand for agriculture use or be planned for later on use.

\section{MATERIALS AND METHODS}

The studied area lies at the north-western outskirt of El Fayoum depression, adjacent to Qaroun Lake between latitudes of 29 o $19^{\prime \prime}$ and $29^{\circ} 26^{\prime \prime} \mathrm{N}$ and longitudes of $30^{\circ} 24^{\prime \prime}$ and $30^{\circ} 34^{\prime \prime} \mathrm{E}$, within the arid desert belt and characterized by the continental climate, Figure (1). 


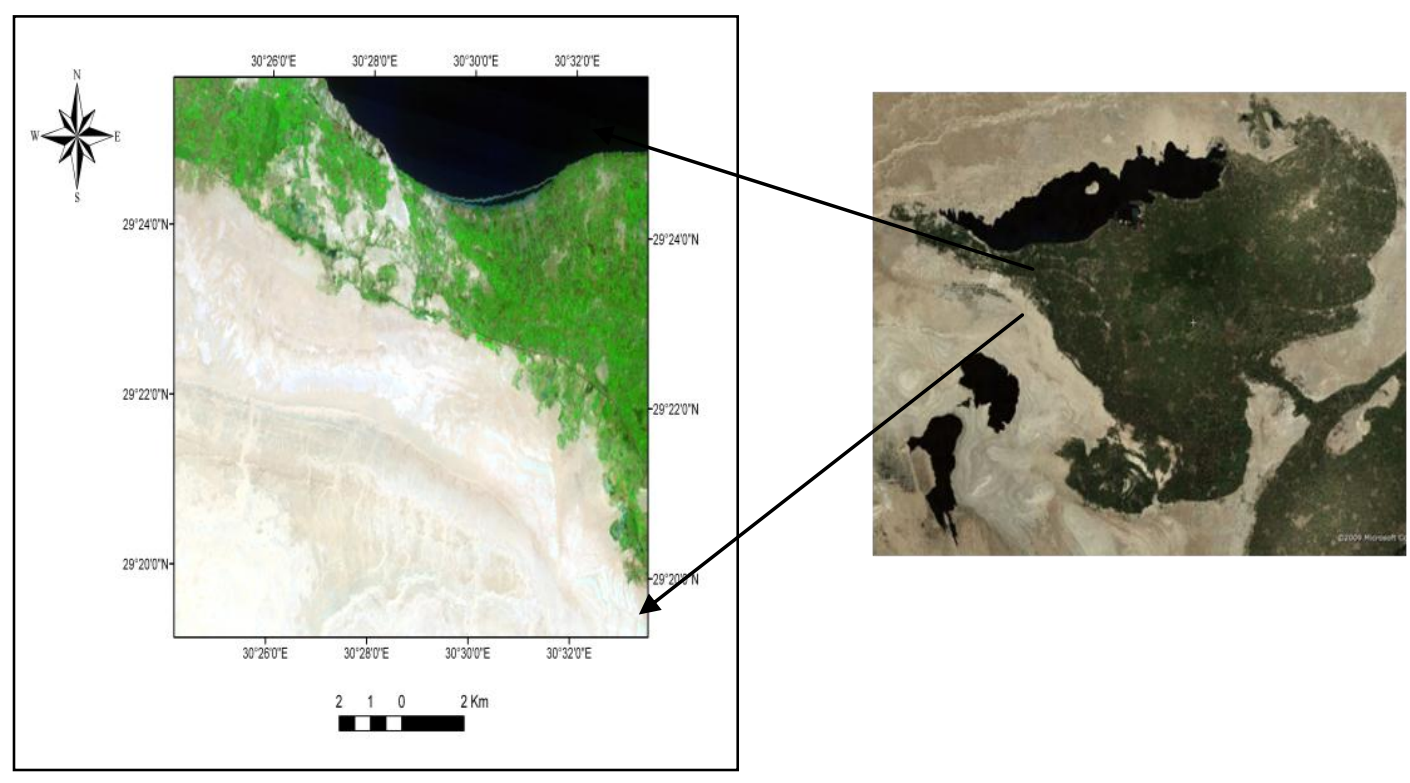

Figure (1); location of the study area.

Satellite image interpretation:

Data: Earth Observation data were used for this study: the Landsat TM 5 and 7 Images were acquired in 1984 and 2005 respectively. The Landsat images had a spatial resolution of $30 \mathrm{~m}$. Auxiliary information was obtained through field surveys. GPS was used to collect Ground Control Points and ground truth data pertaining to the different land use classes. The digital interpretation of the satellite data was performed using the following software:

Erdas Imagine 9.1: Image pre-processing and accuracy assessment.

ENVI 4.7: Spectral Angle Mapper (SAM) approach.

ARCGIS 9.3: Design the data base, layout of maps.

Image Pre-processing: Before the classification process, Landsat images were geometrically corrected, Atmospheric correction (FAASH using ENVI software) and spectrally enhanced. The Landsat data was georeferenced to UTM zone $36 \mathrm{~N}$, with the datum WGS 1984.

Image Classification: To get information about the land use and land cover inventory in the study area the classification methods was preformed based on both Spectral Angle Mapper (SAM) approach, which bears the potential to classify pixels not only based on their spectral information, but also by their texture and local context. The results of the classification method was discussed and compared and an accuracy assessment was performed. The accuracies were determined through a pixel to pixel comparison and expressed as overall, producer, user and in class accuracy (Congalton, 1991 and Story and Congalton, 1986).

Spectral Angle Mapper (SAM): The Spectral Angle Mapper (Yuhas, et al., 1992) is a technique to classify hyperspectral data by determining the similarity between an endmember spectrum and a pixel spectrum in a n-dimensional space. This technique can also be used for multispectral data sets. Smaller angles represent closer matches to the reference spectrum. Since this method uses only the direction of a vector and

Fayoum J. Agric. Res. \& Dev., Vol.25, No.1, January, 2011 
not its length, it is insensitive to illumination and albedo effects. Image-based endmember spectra of the land use types in the study area were used as input for Spectral Angle Mapper classification. For the SAM classification a threshold (rang between 0.05 and 0.40 radian) based on the spectral angle was set. Pixels with an angle larger than this value were not classified. To identify image-derived endmember, Pixel Purity Index (PPI) algorithms were performed on the TM data sets; these spectra were associated to specific land use classes and used to perform the Spectral Angle Mapper classification.

Post Classification Comparison Change Detection: The Post-Classification comparison change detection was done after classifying the rectified images separately from the two time periods (1984 and 2005). The classified images were then, compared and analyzed using ENVI software to create the change-detection matrix and construct change maps.

\section{Field work:}

After the interpretation of satellite images (2005), reconnaissance survey tracks were planned to cross the majority of the different mapping units and to cover the significant land features that occur in the area. Thirty-two mini pits were located and studied for setting up the physiographic boundaries and characteristic of soil map legend. The sample areas were selected so that they cut across the different mapping units and the significant features observed in the area. Eighteen soil profiles were examined along the different sample areas. Detailed morphological description was recorded for each of the studied soil profiles, on the bases outlined by USDA (2003) and classified according to USDA (1975) and (2010).

Re-Interpretation satellite images:

Detailed re-interpretation analysis was done, on satellite image, to finalize the interpretation boundaries after the establishment of the ground truth in the field. A comparison was done to recognize and compare the different landcover landuse in reality and the corresponding appearance on the TM image and on the aerial photographs, the collected information was used later for the establishment of the landcover landuse legend phases. Map legend was finalized and the physiographic units of the aerial photos were finally translated in terms of soils, the finalized tabular legend was constructed with the help of terminology instructions given by, Zinck (1989).

\section{Laboratory analysis:}

A total of 46 disturbed and undisturbed soil samples were collected ,air dried, crushed, sieved through a $2 \mathrm{~mm}$ sieve to obtain the fine earth used for physical and chemical analysis. The elements of soil color description, i.e., the colour name and notations were determined using the Munsell Soil Colour Chart (1975). Particle size distribution was carried out according to Gee and Bander (1986) using sodium hexameta phosphate as dispersing agent. Some soil physical and chemical analyses were carried out according the outlines of Page $\boldsymbol{e t}$ al. (1982) and Klute (1986). Soils under investigation were evaluated using the parametric system undertaken by Sys and Verheye (1978).

\section{RESULTS AND DISCUSSIONS:}

1- Land cover/land changeS detection: a. Spectral Angle Mapper (SAM) Image Analysis:

As previously mentioned, four land use \&land cover classes (Urban, Agriculture, Water and Desert) on the studied area were defined and classified using the Spectral Angle Mapper (SAM). Thematic maps were created for each of the satellite images considered in this study. Figures ( 2 and 3) and table (1) show

Fayoum J. Agric. Res. \& Dev., Vol.25, No.1, January, 2011 
the maps established from the Spectral Angle Mapper (SAM) for Landsat TM images in 1984 and 2005 respectively.

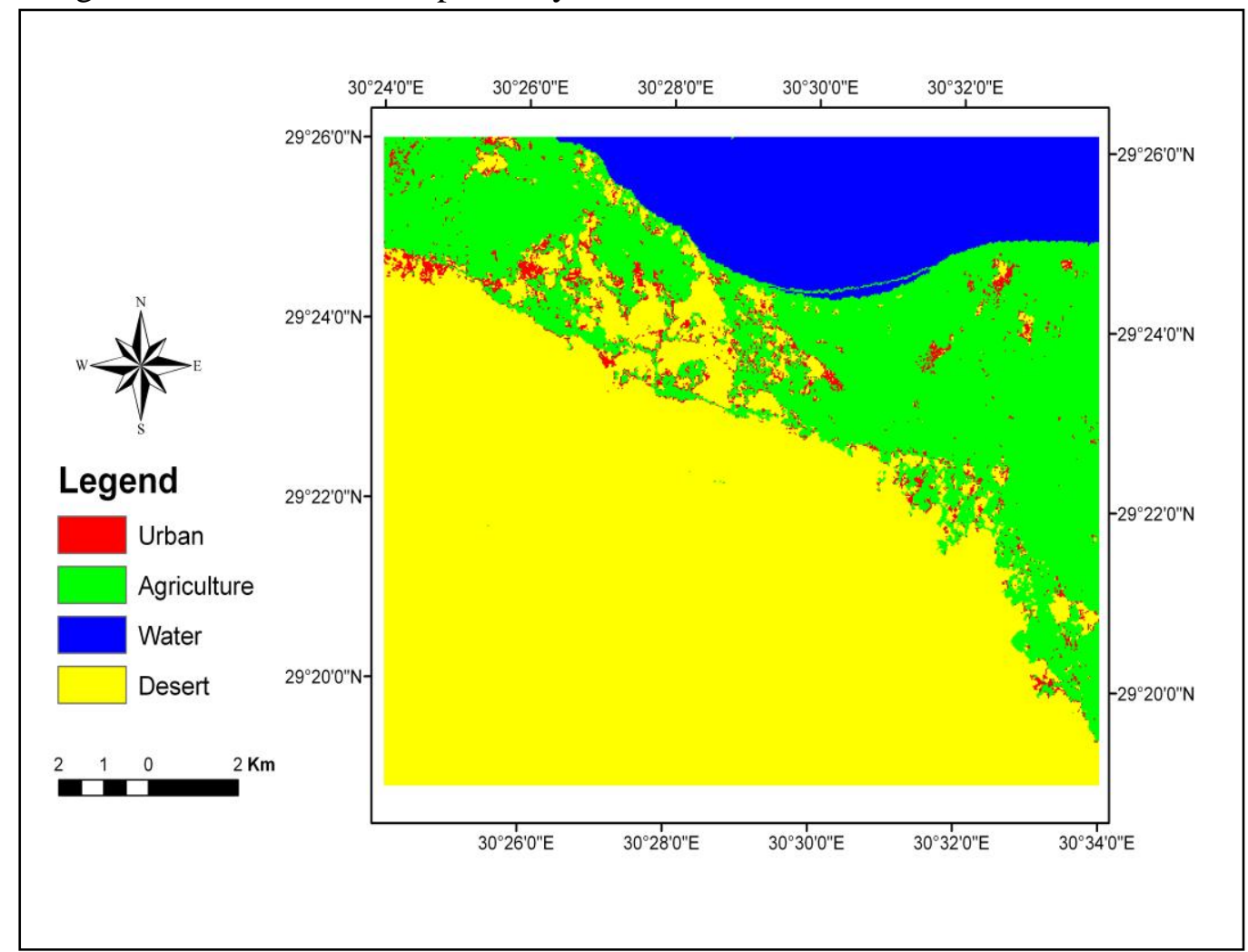

Figure (2): Land use/ land cover map (1984).

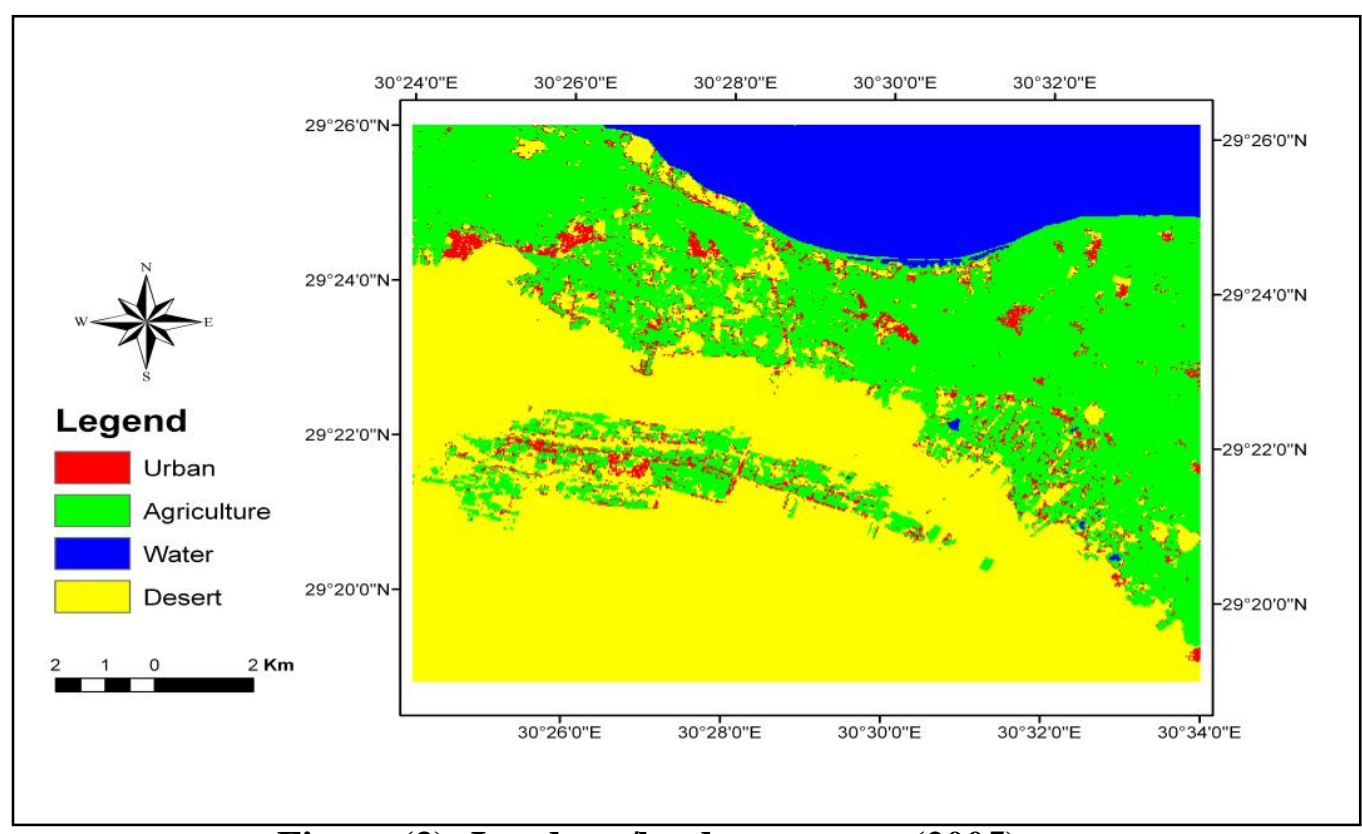

Figure (3): Land use/land cover map (2005).

Fayoum J. Agric. Res. \& Dev., Vol.25, No.1, January, 2011 
MONITORING LAND COVER CHANGES OF THE NORTH-WESTERN....

Table (1): Land use/land cover and area changes in hectare and \%.

\begin{tabular}{|c|c|c|c|c|c|c|}
\hline $\begin{array}{c}\text { Land use/land } \\
\text { cover }\end{array}$ & \multicolumn{2}{|c|}{ Area at1984 } & \multicolumn{2}{c|}{2005} & \multicolumn{2}{c|}{$\begin{array}{c}\text { Area changes from } \\
1984 \text { to 2005 }\end{array}$} \\
\hline & Fed & $\%$ & Fed & $\%$ & Fed & $\%$ \\
\hline Urban & $\mathbf{1 2 1 2 . 4 9}$ & 2.29 & $\mathbf{1 9 8 2 . 4 0}$ & $\mathbf{3 . 7 5}$ & $\mathbf{7 6 9 . 7 3}$ & $\mathbf{1 . 4 6}$ \\
\hline Water & $\mathbf{6 8 0 5 . 3 3}$ & $\mathbf{1 2 . 8 7}$ & $\mathbf{6 8 8 3 . 5 4}$ & $\mathbf{1 3 . 0 4}$ & $\mathbf{7 8 . 1 8}$ & $\mathbf{0 . 2 2}$ \\
\hline Agriculture & $\mathbf{1 3 6 4 5 . 9 7}$ & $\mathbf{2 5 . 8 1}$ & $\mathbf{1 7 9 3 3 . 9 1}$ & $\mathbf{3 3 . 9 7}$ & $\mathbf{4 2 8 7 . 9 1}$ & $\mathbf{8 . 1 5}$ \\
\hline Desert & $\mathbf{3 1 1 9 2 . 8 8}$ & $\mathbf{5 9 . 0 1}$ & $\mathbf{2 5 9 8 4 . 5 9}$ & $\mathbf{4 9 . 2 2}$ & $\mathbf{- 5 2 0 7 . 3 4}$ & $\mathbf{- 9 . 7 8}$ \\
\hline
\end{tabular}

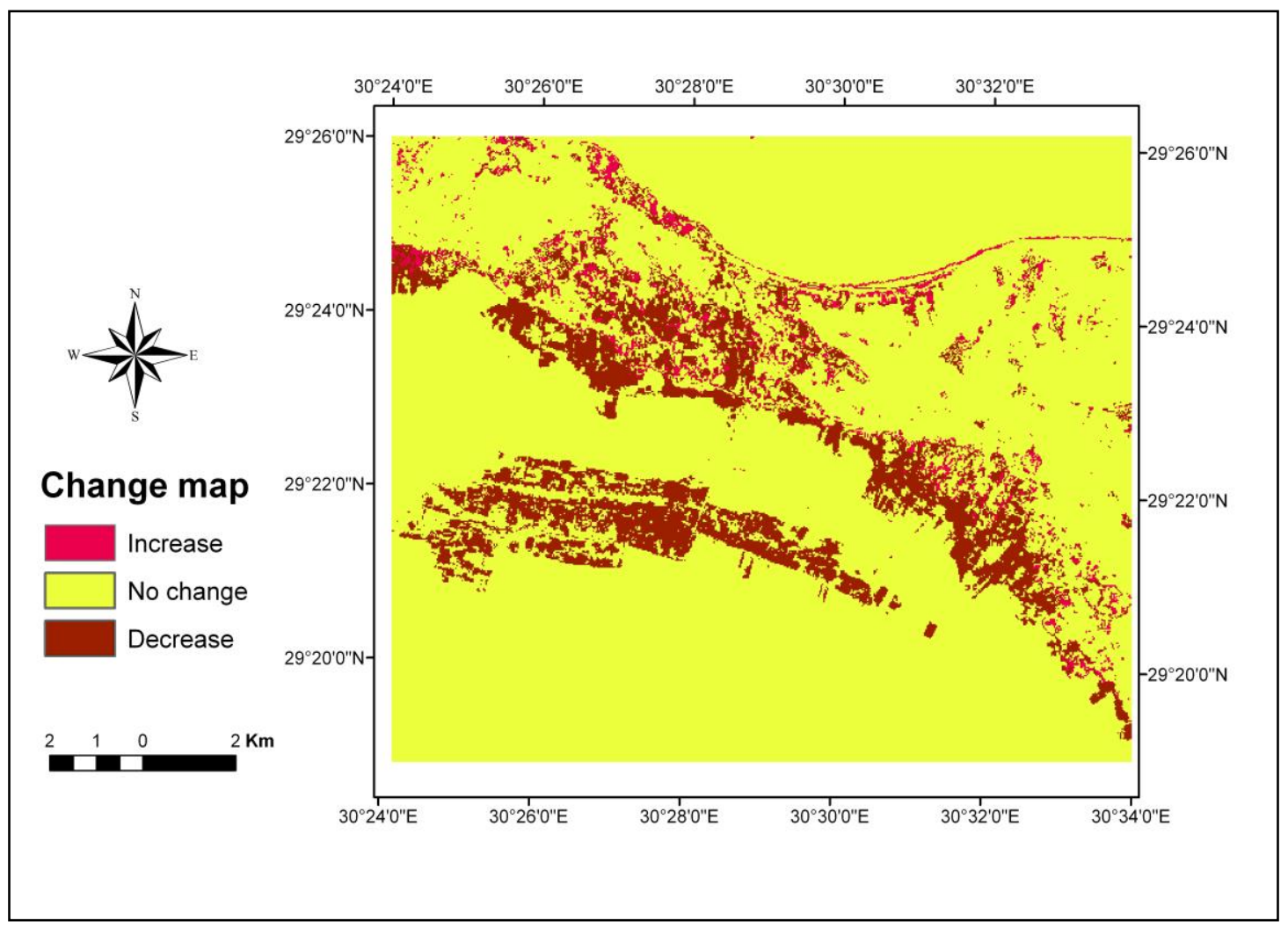

Figure (4): Land use and land cover changes map from 1984 to 2005.

Changes in the land cover were unidirectional for all land use and land cover, Fig. (4), the absolute rates of change for desert decreased during the entire studied period (1984-2005). The decrease in this land use class is as a result of a corresponding increase in agriculture and urban areas. Agriculture increases from $25.817 \%$ to 33.975 during the twenty one years (21 years) period probably due to government's program for the reclamation of new agricultural land. The proportion of urban areas increased from nearly $2.294 \%$ to $3.756 \%$ mainly as a result of population growth. Water increased by 12.875 to $13.041 \%$. The increase of water bodies may be rendered mainly to fish cultivation in big pools adjacent to Qaroun lake shoreline and also may be that corresponding canals on the reclaimed land possibly led to an increase in water bodies. Desert decreased from 59.014 to $49.229 \%$. Conversation the reclaimed land to other land use types agriculture and settlement explains the decrease of desert.

Fayoum J. Agric. Res. \& Dev., Vol.25, No.1, January, 2011 
II. Assissment of Land Physiography:

Physiographic units were delineated in Fig. (5) and their legend has been set up as shown in Table (2), associated with the morphological description of the representative soil profiles, Table (3). The studied area comprised the north-western shoulder of El-Fayoum depression with alluvium and lacustrine parent materials and their natural extension towards the desert with limestone formations. So, the area is rich with alternative sets of interferences from these deposits. The identified physiographic-soil units were delineated in two main formations, i.e. the Nile alluvial plain deposits (flat or almost flat Nile alluvial terraces in different elevations) and desert pediplain which includes (elongated hills, toe slopes, foot slopes, and terraces in different elevations beside to depressed slopes and depression bottom units.

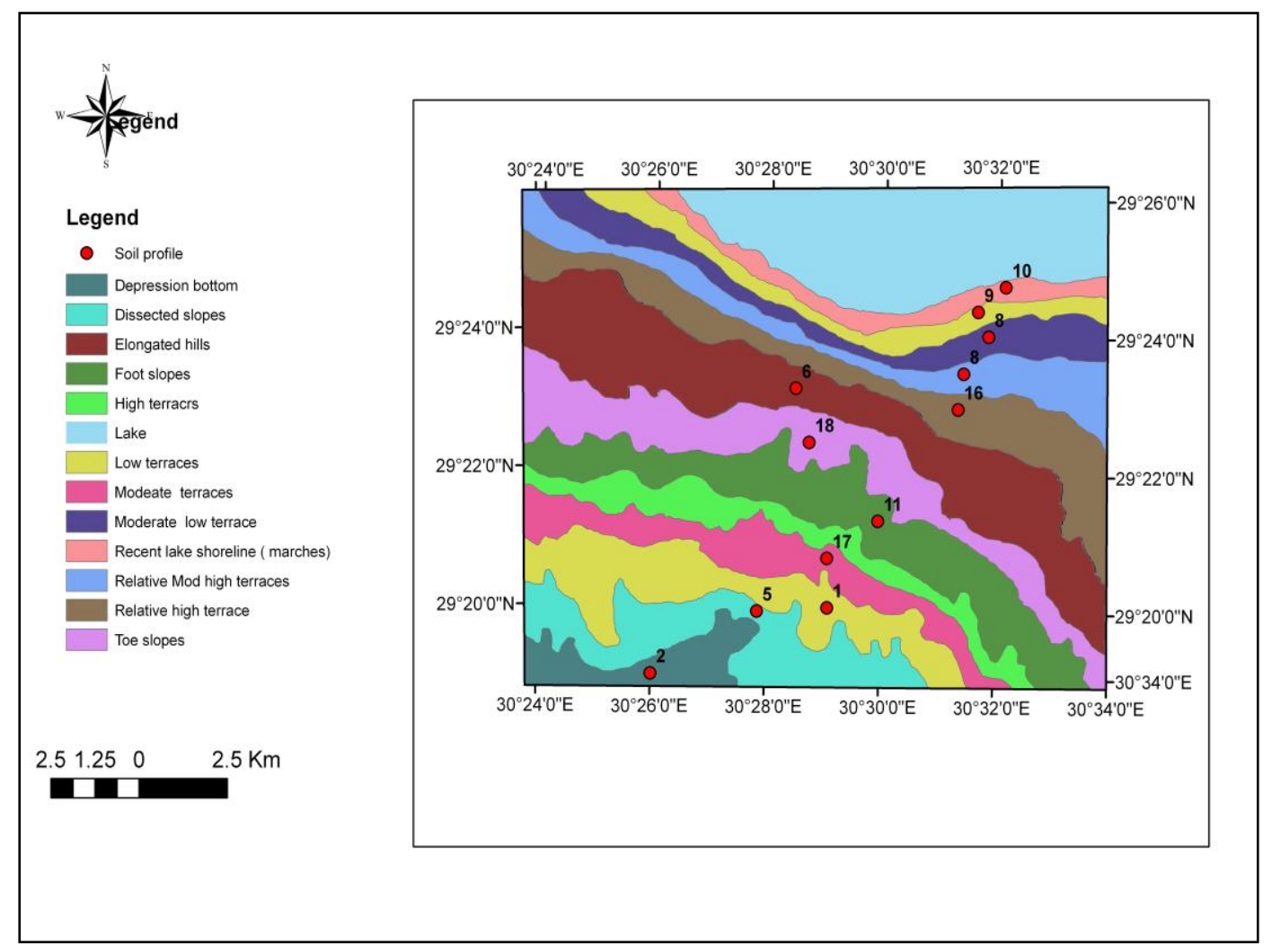

Figure (5): Physiographic units of the studied area

Fayoum J. Agric. Res. \& Dev., Vol.25, No.1, January, 2011 
Table (2): Physiographic units, soil legend and the corresponding areas.

\begin{tabular}{|c|c|c|c|c|c|c|c|}
\hline \multirow{2}{*}{ 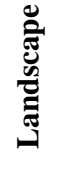 } & \multirow{2}{*}{ 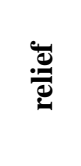 } & \multirow{2}{*}{ } & \multirow{2}{*}{ Landform } & \multirow{2}{*}{$\begin{array}{l}\text { Map } \\
\text { unit }\end{array}$} & \multirow{2}{*}{ 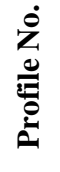 } & \multicolumn{2}{|c|}{ Area } \\
\hline & & & & & & Fed & $\%$ \\
\hline \multirow{8}{*}{ } & \multirow{4}{*}{$\stackrel{\mathscr{\leftrightarrow}}{: 0}$} & \multirow{8}{*}{$\begin{array}{c}\text { Eocene } \\
\text { limestone }\end{array}$} & Elongated hills & pp 111 & 6 & 7929.22 & 15.02 \\
\hline & & & Toe slopes & pp 112 & 3 & 4905.40 & 9.29 \\
\hline & & & Foot slopes & pp 113 & 11 & $\mathbf{5 2 3 9 . 0 8}$ & 9.92 \\
\hline & & & High terraces & pp 211 & 13 & 2429.80 & 4.62 \\
\hline & \multirow{4}{*}{ 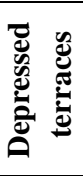 } & & Moderately terraces & pp 212 & 17 & 2955.23 & 5.60 \\
\hline & & & Low terraces & pp 213 & 1 & 3940.61 & 7.46 \\
\hline & & & Dissected slopes & pp 214 & 5 & 3553.25 & 6.73 \\
\hline & & & Depression bottom & pp 215 & 2 & 1566.18 & 2.96 \\
\hline \multirow{5}{*}{$\begin{array}{l}\text { 恶 } \\
\text { 晋 } \\
\text { 总 }\end{array}$} & \multirow{5}{*}{ 递 } & Alluvium & Relatively high terraces & P1 111 & 16 & 4192.39 & 7.95 \\
\hline & & F. lacustrine & $\begin{array}{c}\text { Relatively mod. High. } \\
\text { terraces }\end{array}$ & P1 112 & 18 & 2966.44 & 5.63 \\
\hline & & \multirow{2}{*}{ Alluvium } & Moderately low terraces & P1 113 & 8 & 2576.01 & 4.88 \\
\hline & & & Low terraces & P1 114 & 9 & 1838.33 & 3.48 \\
\hline & & F. lacustrine & Recent lake shoreline & P1 116 & 10 & 1534.96 & 2.91 \\
\hline
\end{tabular}

F. lacustrine =Fluvio-lacustrine

Thus, an injection was occurred between fluvial deposits and local lacustrine ones, which were richer in secondary formations of $\mathrm{CaCO}_{3}$ and gypsum. Said (1990) stated that, in the case of Lake Moeris, where El-Fayoum depression fell completely with the fresh Nile water, that reaches in undate its flood basin. Due to the depositional processes rather than erosional processes, hence the Nile suspended matter was deposited in different physiographic-soil units from the seasonal and periodic flooding. Discharge that is fully confined to El-Fayoum basin maintains high competence, and when discharge exceeds basin capacity, there is a dramatic increase in cross-sectional area associated with expansion into the flood basin. This physiographic-soil unit comprised an area of 13106 fed and includes many land forms which attain graded terraces from high to low terraces and marches. This physiographic unit was represented by soil profiles Nos. 8,9,10,16 and 18 which are deep contain low amounts of $\mathrm{CaCO}_{3}$ in the alluvial deposits of profile16 and, moderately high amounts through fluvio-lacustrine deposits of profiles $8,9,10$ and 18 (Tables 3,4 and 5) .

\section{a. Depressed plain:}

Such depressed plain includes soils of heavy-textured materials, which are subjected to the swelling and shrinkage processes, fitting the requirement to be Vertisols of the Nile alluvial very fine sediments for soil profile No. 8 and saline feature that not adequate to shift the taxonomic unit to be Halic but stell Typic Hapolcalcids for profile (10).

Fayoum J. Agric. Res. \& Dev., Vol.25, No.1, January, 2011 
Tolba S. Abdel Aal \& Abdel Aziz B.A. Belal

Table (3): Morphological description of the studied physiographic units.

\begin{tabular}{|c|c|c|c|c|c|c|c|c|c|}
\hline 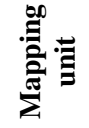 & $\begin{array}{c}\text { Slope } \\
\text { gradient }\end{array}$ & 递定 & $\begin{array}{c}\text { Soil } \\
\text { Parent } \\
\text { material }\end{array}$ & $\begin{array}{c}\text { Soil } \\
\text { Depth } \\
\text { (cm) }\end{array}$ & 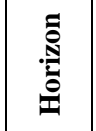 & Soil color & $\begin{array}{c}\text { Modified } \\
\text { Texture } \\
\text { class }\end{array}$ & $\begin{array}{c}\text { Soil } \\
\text { structure }\end{array}$ & $\begin{array}{c}\text { Soil } \\
\text { consistency }\end{array}$ \\
\hline \multirow{3}{*}{ Pu 21} & \multirow{3}{*}{ Sloping } & \multirow{3}{*}{1} & \multirow{3}{*}{$\begin{array}{c}\text { Eocene } \\
\text { limestone }\end{array}$} & $0-20$ & Ap & $2.5 Y 6 / 4$ & G S & Single $\mathbf{g}$ & Loose \\
\hline & & & & $20-60$ & C1 & $2.5 \times 6 / 4$ & ExG SL & Massive & Friable \\
\hline & & & & $60-80$ & $\mathrm{C} 2$ & $2.5 Y 8 / 2$ & ExG LS & Massive & V hard \\
\hline \multirow{3}{*}{ Pu 42} & \multirow{3}{*}{ Nearly level } & \multirow{3}{*}{2} & \multirow{3}{*}{$\begin{array}{c}\text { Aeolian } \\
\text { over } \\
\text { alluvium } \\
\end{array}$} & $0-25$ & Ap & 10YR 6/4 & SL & Massive & Slight. hard \\
\hline & & & & $25-55$ & C & $2.5 Y 8 / 2$ & LS & Massive & Slight. hard \\
\hline & & & & 55-150 & $2 \mathrm{C}$ & 10YR 7/1 & C & Massive & hard \\
\hline \multirow{3}{*}{ Pu 24} & \multirow{3}{*}{$\begin{array}{c}\text { Gently } \\
\text { undulating }\end{array}$} & \multirow{3}{*}{3} & \multirow{3}{*}{$\begin{array}{l}\text { Aeolian - } \\
\text { limestone }\end{array}$} & $0-25$ & Ap & 10YR 5/4 & SL & Massive & Slight. hard \\
\hline & & & & $25-50$ & $\mathbf{C y}$ & 10YR 6/4 & $\mathbf{S}$ & Single $\mathrm{g}$ & Loose \\
\hline & & & & $50-150$ & Cky & 10YR & G S & Single $\mathrm{g}$ & Loose \\
\hline \multirow{3}{*}{ Pu 23} & \multirow{3}{*}{ Sloping } & \multirow{3}{*}{4} & \multirow{3}{*}{$\begin{array}{c}\text { Eocene } \\
\text { limestone }\end{array}$} & $0-25$ & Ap & $2.5 Y 6 / 4$ & VG SL & Massive & Slight. hard \\
\hline & & & & $25-50$ & Ck1 & $2.5 Y 6 / 4$ & ExG LS & Single $\mathrm{g}$ & Loose \\
\hline & & & & $50-70$ & Ck2 & $2.5 Y 7 / 4$ & ExG S & Single $\mathrm{g}$ & Loose \\
\hline \multirow{2}{*}{ Pu31 } & \multirow{2}{*}{$\begin{array}{c}\text { Gently } \\
\text { undulating }\end{array}$} & \multirow{2}{*}{5} & \multirow{2}{*}{$\begin{array}{l}\text { Aeolian- } \\
\text { limestone }\end{array}$} & $0-20$ & Cky1 & $2.5 Y 6 / 4$ & G SL & Massive & Slight. hard \\
\hline & & & & $20-60$ & Cky2 & $2.5 Y 7 / 4$ & ExGLS & Massive & hard \\
\hline Pu 12 & \multirow{3}{*}{ Almost flat } & 6 & & 0-30 & Ckyz1 & $2.5 Y 7 / 6$ & ExG LS & Single $\mathbf{g}$ & Loose \\
\hline Pu 11 & & 7 & limestone & $\mathbf{0 - 3 0}$ & Cky1 & $2.5 Y 7 / 4$ & ExG SL & Massive & Friable \\
\hline & & & & $30-60$ & Cky2 & $2.5 Y 7 / 4$ & ExG LS & Single $\mathbf{g}$ & Loose \\
\hline & & & Alluvium & $\mathbf{0 - 3 0}$ & Ap & 10YR 4/4 & CL & Massive & Slight. hard \\
\hline Pl 13 & undulating & 8 & and old & $30-60$ & C1 & 10YR 5/3 & CL & M m sbk & Hard \\
\hline & & & lacustrine & $60-150$ & $2 \mathrm{C}$ & 10YR 5/2 & C & M m sbk & V. hard \\
\hline & & & & $\mathbf{0 - 3 0}$ & Apk & 10YR 6/4 & SCL & Massive & Hard \\
\hline PI 12 & Almost flat & 9 & & $30-60$ & C1 & 10YR 7/3 & $\mathbf{C}$ & M m sbk & V. hard \\
\hline & & & & $60-150$ & $\mathrm{C2}$ & 10YR 6/4 & SCL & M m sbk & Hard \\
\hline & & & & 0-30 & Apk & 10YR 5/3 & CL & Massive & Slight. hard \\
\hline PI 14 & undulating & 10 & $\begin{array}{l}\text { Fiuvio- } \\
\text { lacustrine }\end{array}$ & $30-60$ & Ckz1 & 10YR 6/2 & CL & Massive & Slight. hard \\
\hline & & & & 60-120 & $2 \mathrm{Ck}$ & 10YR 6/2 & $\mathbf{C}$ & Massive & V hard \\
\hline & & & & 0-30 & Apky & 10 YR $6 / 3$ & SCL & Massive & hard \\
\hline Pu 41 & Almost flat & 11 & colluxium- & $30-50$ & Cky1 & $2.5 Y 5 / 4$ & G SL & Massive & Friable \\
\hline & & & & 50-150 & Cky2 & $10 Y r 7 / 3$ & G LS & Single $\mathrm{g}$ & Loose \\
\hline Pu 22 & Almost flat & 12 & & 0-30 & Cky & $2.5 Y 7 / 4$ & VG LS & Massive & V hard \\
\hline Pu 32 & Sloping & 13 & Eocene & $0-30$ & $\mathbf{C k}$ & $2.5 Y 7 / 4$ & G LS & Single g & Loose \\
\hline 1002 & Diopmy & 10 & limestone & $30-60$ & Cky2 & $2.5 Y 6 / 2$ & ExG LS & Massiv e & Loose \\
\hline PI 21 & Almost flat & 14 & & $0-25$ & Ckyz1 & $2.5 Y 7 / 4$ & SCL & Massive & V hard \\
\hline & Amiost Hat & 14 & & $25-40$ & Ckyz2 & $2.5 Y 7 / 4$ & ExG SC & Massive & V hard \\
\hline Pu 33 & Nearly level & 15 & & 0-30 & Cky1 & $2.5 Y 5 / 3$ & G LS & Single $\mathbf{g}$ & Loose \\
\hline & & & & $30-60$ & Cky2 & $2.5 Y \quad 6 / 4$ & G SL & Massive & Slight. hard \\
\hline & & & & 0-30 & Ap & 10YR 5/3 & SCL & Massive & Slight. hard \\
\hline PI 11 & Nearly level & 16 & Alluvium & $30-60$ & C1 & 10YR 6/3 & SCL & M m sbk & Hard \\
\hline & & 10 & Anuviumi & $60-90$ & $\mathrm{C2}$ & 10YR 8/3 & SCL & Massive & V hard \\
\hline & & & & $90-150$ & $\mathrm{C3}$ & 10YR 7/3 & $\mathbf{C}$ & M m sbk & Hard \\
\hline & & & & 0-30 & Ap & 10YR 4/3 & SCL & Massive & Hard \\
\hline PI 16 & undulating & 17 & $\begin{array}{l}\text { Fiuvio- } \\
\text { lacustrine }\end{array}$ & $30-60$ & Ck1 & 10YR 5/4 & SC & M m sbk & Hard \\
\hline & & & & 60-110 & Ck2 & 10YR 6/4 & $\mathbf{L S}$ & Massive & Hard \\
\hline & & & & 0-30 & Ap & $10 Y R 4 / 2$ & SCL & Massive & Hard \\
\hline Pl 15 & Nearly level & 18 & alluvium & $30-60$ & C1 & 10YR 6/3 & $\mathbf{L S}$ & Massive & Hard \\
\hline & & & & $60-150$ & $2 \mathrm{C}$ & $2.5 Y 6 / 2$ & $\mathbf{S}$ & Single $\mathbf{g}$ & Loose \\
\hline
\end{tabular}

$\mathrm{S}=$ Sand, $\mathrm{LS}=\mathrm{Loamy}$ sand, $\mathrm{SL}=$ Sandy loam, $\mathrm{SCL}=$ Sandy clay loam, $\mathrm{CL}=$ Clay loam and $\mathrm{C}=\mathrm{Clay}, \mathrm{G}=$ Gravel:VG=Very gravelly, and ExG= Excessively Gravelly Mmsbk=Medium moderate subangular blocky, Cstsbk=Coarse strong subangular blocky.

Fayoum J. Agric. Res. \& Dev., Vol.25, No.1, January, 2011 
b. Pediplain (pp):

This physiographic unit represents the biggest unit in the studied area, and its representative soils occupy an area of about 32700 fed. These soils recognized by quite clear relief and the light greytone according to the soil surface cover (limestone fragments and blown sands). According to the surface features of this unit, it could be categorized into nine landforms, i.e., elongated hills, toe slopes, foot slopes, and many graded terraces beside to depressed slopes and bottom. This unit extends from the depression western side with a parallel direction to its border towards Birket Qaroun at the southern part. The soils developed on this unit represent the foot-slope of the rock structure, the disintegrated weathered rock fragments, surface pavements, and different formations of limestone, marl and sand cover. The representative soil profiles have different soil depths varied from deep for soil profile Nos. 2, 3 and 11 to moderate for soil profile Nos.1, 4, 5, 7, 13 and 15 and shallow for soil profile Nos. 6 and 12.

The high content of gravel (lime fragments) associated with the disintegration of limestone bedrock and their contents increased with shallow soil depths, Tables (3, 4 and 5). The deep soils of toe slopes, and terraces landform units characterized by the depositional deposits of sands and weathered limestone within some of coarse materials. The representative area has been artificially modified in order to change the landscape into depressed terraces to suite-irrigated agriculture soils, these units have topographic landscape ranges between almost flat to gently undulating. The soils of these units are represented by soil profile Nos. 3, 4, 11, 13, 14 and 17. The elongated hill unit covers an area of about 7929 fed and located at the south-western side of the studied area (pp 111) has shallow depth and gravelly coarse texture and was represented by soil profile Nos. 6 and 7 .

Its elevation reaches $17-20 \mathrm{~m}$ above sea level forming ridge area. It is also occurred as barren limestone (original structure) outcrops with a gently sloping surface associated with the rolling slope. The toe slopes (pp112) unit is more deep soil (profile Nos. 3 and 4), also the foot slope unit (pp 113) of profile No. 11.

Fayoum J. Agric. Res. \& Dev., Vol.25, No.1, January, 2011 
Tolba S. Abdel Aal \& Abdel Aziz B.A. Belal

Table (4): Some physico- chemical properties of the studied soil profiles.

\begin{tabular}{|c|c|c|c|c|c|c|c|c|c|c|c|c|}
\hline \multirow{2}{*}{$\begin{array}{l}\dot{\theta} \\
\dot{0} \\
\dot{0} \\
\dot{0}\end{array}$} & \multirow{2}{*}{ 营 } & \multirow{2}{*}{ 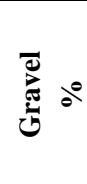 } & \multicolumn{4}{|c|}{ Particle size distribution \% } & \multirow{2}{*}{ 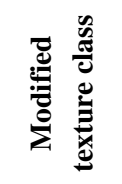 } & \multirow{2}{*}{ 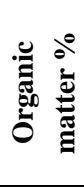 } & \multirow{2}{*}{$\begin{array}{l}0 \\
0 \\
0 \\
0 \\
0\end{array}$} & \multirow{2}{*}{ 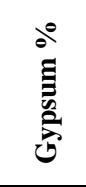 } & \multirow{2}{*}{ 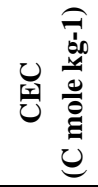 } & \multirow{2}{*}{ 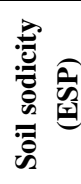 } \\
\hline & & & 芯 & 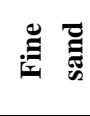 & 言 & $\overrightarrow{\mathrm{C}}$ & & & & & & \\
\hline \multirow{3}{*}{1} & $0-20$ & 31.20 & 52.37 & 36.19 & 7.21 & 4.23 & GS & 1.04 & 12.47 & 6.21 & 5.11 & 6.34 \\
\hline & $20-60$ & 68.20 & 37.37 & 29.33 & $\mathbf{1 4 . 8 5}$ & $\mathbf{1 8 . 4 5}$ & Ex G SL & 0.13 & $\mathbf{1 0 . 7 5}$ & 4.36 & 16.67 & 7.58 \\
\hline & $60-80$ & 70.30 & 44.36 & & & & X G LS & 0.13 & 11.52 & 5.61 & 9.37 & 7.22 \\
\hline \multirow{3}{*}{2} & $0-25$ & 4.50 & 34.68 & & & & & & 4.94 & 2.21 & 11.53 & \\
\hline & $25-55$ & 0.00 & 37.56 & & 9.81 & & $\mathbf{L S}$ & 0.16 & 8.54 & 1.33 & 8.94 & \\
\hline & $55-150$ & $\mathbf{0 . 0 0}$ & 11.04 & 29.32 & 8.31 & 48.65 & $\mathbf{C}$ & 0.12 & 6.97 & 1.35 & 46.75 & 6.84 \\
\hline \multirow{3}{*}{3} & $0-25$ & 5.00 & 36.34 & 39.25 & 11.36 & $\mathbf{1 3 . 0 5}$ & SL & 0.64 & 8.02 & 6.28 & 15.76 & 4.59 \\
\hline & $25-50$ & 5.11 & 38.36 & 51.46 & 4.82 & 5.36 & $\mathbf{S}$ & 0.16 & 11.38 & 7.14 & 6.27 & 5.27 \\
\hline & 50-150 & 18.32 & 40.25 & 51.45 & 3.69 & 4.61 & G S & 0.11 & 16.56 & 7.65 & 4.18 & 4.96 \\
\hline \multirow{3}{*}{4} & $0-25$ & 48.34 & 28.70 & 42.34 & 10.57 & 18.39 & VG SL & 0.85 & 4.01 & 4.91 & 21.87 & 7.35 \\
\hline & $25-50$ & 52.98 & 38.96 & 40.48 & 11.05 & 9.51 & Ex G LS & 0.44 & 5.43 & 3.21 & 9.42 & \\
\hline & $50-70$ & 68.04 & 48.37 & 41.61 & 3.91 & 6.11 & Ex GS & 0.11 & 5.68 & 4.24 & 7.57 & 6.24 \\
\hline \multirow{2}{*}{5} & $0-20$ & 31.1 & 36.98 & & 12.05 & 11.33 & G SL & 0.53 & 17.63 & 13.21 & 13.24 & \\
\hline & $20-60$ & 74.64 & 39.19 & 42.87 & 12.53 & 5.41 & Ex LS & 0.28 & 28.71 & 16.25 & 7.53 & 5.21 \\
\hline 6 & $0-30$ & 73.10 & 52.92 & 28.48 & & 23 & Ex LS & 0.12 & 16.53 & 10.82 & 8.643 & .54 \\
\hline \multirow{2}{*}{7} & $0-30$ & 63.54 & 24. & 40. & 16. & 18.37 & Ex G SL & 0.85 & 16.35 & 6.22 & 20.14 & \\
\hline & $30-60$ & 68.43 & 39.35 & 36.76 & 11.08 & 12.81 & Ex G LS & 0.33 & 23.22 & 7.17 & 13.28 & 7.94 \\
\hline \multirow{3}{*}{8} & 0-30 & 0.00 & 12.32 & 18.09 & 28.91 & 68 & CL & 0.96 & 7.46 & 1.24 & 40.51 & 0.14 \\
\hline & $30-60$ & 0.00 & 9.82 & 18.39 & 30.11 & 41.68 & $\mathbf{C}$ & 0.65 & 9.11 & 0.96 & 43.36 & 11.32 \\
\hline & $60-150$ & 0.00 & & 17 & & & $\mathbf{C}$ & & 11.48 & 3.11 & 48.21 & .56 \\
\hline \multirow{3}{*}{9} & 0-30 & 00 & & & & & SCL & & 10.3 & 1.24 & 0.45 & .34 \\
\hline & $30-60$ & 0.00 & 7 & 23.5 & 6.97 & 42.28 & $\mathbf{C}$ & 1 & 8.85 & 0.52 & 40.72 & 2.64 \\
\hline & $60-150$ & 0.00 & 12.37 & 45. & 17.38 & 24.35 & SCL & 0.65 & 6.72 & 0.95 & 26.64 & 10.07 \\
\hline \multirow{3}{*}{10} & $\mathbf{0 - 3 0}$ & 0.00 & & & & & & & 10.63 & 3.62 & 33.64 & \\
\hline & $30-60$ & 00 & & & & & CL & & 6.25 & 3.25 & 40.92 & \\
\hline & $60-150$ & 0.00 & & & $\mathbf{1 0}$ & & $\mathbf{C}$ & 0.73 & 13.56 & 2.45 & 46.48 & 10.84 \\
\hline & & 3.22 & & & & & CL & & 8.92 & 7.32 & 38.21 & 8.56 \\
\hline 11 & $30-50$ & 10.13 & 23.66 & & 22 & & G SL & & 15.43 & 15.39 & 14.36 & 7.35 \\
\hline & 50-150 & 28.64 & 28. & 51. & & & G LS & & 16.72 & 11.23 & 8.25 & 7.68 \\
\hline 12 & 0-30 & 52.24 & 42.3 & 40. & 12.84 & 4.12 & VG LS & 0.38 & 31.84 & 7.20 & 5.14 & 9.22 \\
\hline 13 & $0-30$ & 36.71 & & & $\mathbf{1 3 . 4 5}$ & & VG LS & 0.64 & 12.33 & 6.24 & 7.18 & 10.32 \\
\hline & $30-60$ & 67.24 & & & & & Ex G LS & & 27.69 & 7.32 & 12.24 & 10.94 \\
\hline 14 & $0-25$ & 5.21 & 34.96 & 28. & 2.86 & 23.65 & SCL & 0.84 & 16.28 & 10.85 & 22.11 & 8.94 \\
\hline 14 & $25-40$ & 64.28 & 27.28 & & 13.54 & 39.27 & Ex G SC & 0.14 & 36.12 & 11.32 & 37.39 & 8.71 \\
\hline 1 & $0-30$ & 14.32 & & & & & & & 13.94 & & 6.34 & 7.33 \\
\hline 15 & $30-60$ & 19.97 & 38. & & & & & & 27.54 & 7.25 & 11.48 & 8.14 \\
\hline & 0-30 & 0.00 & & & & & & & 4.95 & 1.23 & 33.51 & 12.35 \\
\hline & $30-60$ & 0.00 & & & & & & 1 & 5.22 & 2.64 & 37.39 & \\
\hline 10 & $60-90$ & 0.00 & 14.40 & 29.55 & 2 & 34.37 & SCL & 0.9 & 3.28 & & 36.24 & 11.35 \\
\hline & $90-150$ & 0.00 & 8.34 & 23.95 & 23.79 & 43.92 & $\mathrm{C}$ & 0.65 & 5.32 & 0.66 & 45.36 & 11.95 \\
\hline & 0-30 & 0.00 & & 44.67 & & & & 1.36 & 6.05 & 1.22 & 36.52 & 12.35 \\
\hline 17 & $30-60$ & & & & & & & & & & 46.37 & \\
\hline & $60-150$ & 0.00 & 27.85 & 55.74 & 7.84 & 8.57 & LS & 0.56 & 17.38 & 2.25 & 7.08 & 12.95 \\
\hline & $0-30$ & 0.00 & 14.16 & 44.07 & 17.66 & 24.11 & SCL & 1.64 & 8.20 & 1.65 & 27.10 & 10.28 \\
\hline 18 & & & & & & & & & 4.92 & & 8.94 & 12.65 \\
\hline & $60-150$ & 0.00 & 38.94 & 51.73 & 3.69 & 5.64 & $\mathbf{S}$ & 0.68 & 6.72 & 2.54 & 4.83 & 11.94 \\
\hline
\end{tabular}

Where: $\mathrm{S}=$ Sand, $\mathrm{LS}=$ loamy sand, $\mathrm{SL}=$ Sandy loam, $\mathrm{SCL}=$ Sandy cay loam, $\mathrm{SC}=$ Sandy clay, $\mathrm{CL}=$ Clay loam, and $\mathbf{C} .=$ Clay.G $=$ Gravelly, VG= Very gravelly Ex G=Extremely gravelly

Fayoum J. Agric. Res. \& Dev., Vol.25, No.1, January, 2011 
Table (5): Chemical analysis of soil paste extract of the studied soil profiles.

\begin{tabular}{|c|c|c|c|c|c|c|c|c|c|c|c|}
\hline \multirow{2}{*}{$\begin{array}{c}\text { Profile } \\
\text { No }\end{array}$} & \multirow{2}{*}{$\begin{array}{c}\text { Depth } \\
\text { (cm) }\end{array}$} & \multirow{2}{*}{$\begin{array}{l}\text { Soil } \\
\text { pH }\end{array}$} & \multirow{2}{*}{$\begin{array}{c}\text { ECe } \\
(\mathrm{dS} / \mathrm{m})\end{array}$} & \multicolumn{4}{|c|}{ Soluble cations ( $m$ mole $\mathbf{L}^{-1}$ ) } & \multicolumn{4}{|c|}{ Soluble anions (m mole $\mathrm{L}^{-1}$ ) } \\
\hline & & & & $\mathrm{Ca}^{2+}$ & $\mathbf{M g}^{2+}$ & $\mathrm{Na}^{+}$ & $\mathbf{K}^{+}$ & $\mathrm{CO}_{3}{ }^{2-}$ & $\mathrm{CO}_{3}{ }^{2-}$ & $\mathrm{Cl}^{-}$ & $\mathrm{SO}_{4}{ }^{2-}$ \\
\hline \multirow{3}{*}{1} & $0-20$ & 7.67 & 3.26 & 10.58 & 6.14 & 14.46 & 0.32 & 31.5 & 0.00 & 14.14 & 17.01 \\
\hline & $20-60$ & 8.01 & 4.28 & 14.28 & 9.14 & 18.47 & 0.41 & 42.3 & 0.00 & 18.20 & 22.41 \\
\hline & $60-80$ & 8.11 & 4.09 & 13.51 & 7.37 & 17.95 & 0.39 & 39.22 & 0.00 & 16.44 & 21.17 \\
\hline \multirow{3}{*}{2} & $0-25$ & 7.51 & 7.88 & 24.96 & 12.65 & 30.84 & 0.66 & 69.11 & 0.00 & 29.39 & 38.70 \\
\hline & $25-55$ & 7.59 & 5.42 & 18.28 & 11.98 & 24.31 & 0.51 & \begin{tabular}{|l|}
55.08 \\
\end{tabular} & 0.00 & 24.39 & 29.74 \\
\hline & $55-150$ & 7.57 & 4.96 & 16.64 & 8.85 & 23.09 & 0.37 & 48.95 & 0.00 & 22.34 & 25.94 \\
\hline \multirow{3}{*}{3} & $0-25$ & 7.54 & 3.24 & 10.92 & 7.12 & 14.08 & 0.29 & 32.41 & 0.00 & 14.25 & 17.54 \\
\hline & $25-50$ & 7.43 & 4.28 & 13.44 & 8.20 & 19.32 & 0.39 & 41.35 & 0.00 & 16.95 & 23.15 \\
\hline & $50-150$ & 7.43 & 5.27 & 16.57 & 11.72 & 23.24 & 0.42 & 51.95 & 0.00 & 22.55 & 28.05 \\
\hline \multirow{3}{*}{4} & $0-25$ & 7.66 & 3.21 & 10.87 & 6.03 & 14.37 & 0.38 & 31.65 & 0.00 & 13.28 & 17.72 \\
\hline & $25-50$ & 7.84 & 3.65 & 12.24 & 7.62 & 15.84 & 0.41 & 36.11 & 0.00 & 16.19 & 18.98 \\
\hline & 50-70 & 7.67 & 4.18 & 13.12 & 8.47 & 18.86 & 0.50 & 40.95 & 0.00 & 18.40 & 21.70 \\
\hline \multirow{2}{*}{5} & $0-20$ & 7.78 & 11.68 & 35.96 & 22.11 & $\mathbf{5 3 . 3 5}$ & 0.94 & $\mathbf{1 1 2 . 3 6}$ & 0.00 & 50.25 & 60.07 \\
\hline & $20-60$ & 7.61 & 12.85 & 39.68 & 25.45 & 58.67 & 1.04 & 124.84 & 0.00 & 55.56 & 66.96 \\
\hline 6 & 0-30 & 8.11 & 67.18 & 224.72 & 132.42 & 301.95 & 3.05 & 662.14 & 0.00 & 313.63 & 344.27 \\
\hline \multirow{2}{*}{7} & $0-30$ & 7.49 & 7.88 & 26.52 & 13.28 & 35.87 & 0.87 & 76.54 & 0.00 & 33.84 & 41.08 \\
\hline & $30-60$ & 7.73 & 9.64 & 31.67 & 16.40 & 45.12 & 0.89 & 94.08 & 0.00 & 39.60 & 52.64 \\
\hline \multirow{3}{*}{8} & $0-30$ & 8.23 & 3.17 & 8.60 & 6.43 & 15.95 & 0.27 & 31.25 & 0.00 & 13.46 & 16.74 \\
\hline & $30-60$ & 8.41 & 2.85 & 7.41 & 6.76 & 14.16 & 0.28 & 28.61 & 0.00 & 12.07 & 15.17 \\
\hline & $60-150$ & 8.12 & 2.90 & 8.29 & 6.33 & 14.05 & 0.29 & 28.96 & 0.00 & 11.81 & 15.02 \\
\hline \multirow{3}{*}{9} & $0-30$ & 8.01 & 8.62 & 24.13 & 20.51 & 38.28 & 0.72 & 83.64 & 0.00 & 37.51 & 44.31 \\
\hline & $30-60$ & 8.12 & 5.18 & 14.25 & 12.63 & 22.95 & 0.64 & 50.47 & 0.00 & 22.55 & 26.24 \\
\hline & $60-150$ & 8.16 & 4.83 & 13.28 & 11.08 & 23.17 & 0.58 & 48.11 & 0.00 & 21.27 & 25.49 \\
\hline \multirow{3}{*}{10} & $0-30$ & 7.87 & 7.15 & 28.32 & 15.80 & 26.38 & 0.46 & 50.96 & 0.00 & 23.65 & 26.47 \\
\hline & $30-60$ & 7.86 & 15.30 & 48.97 & 28.89 & 70.87 & 1.12 & $\mathbf{1 4 9 . 8 5}$ & 0.00 & 66.22 & 81.46 \\
\hline & $60-150$ & 7.92 & 10.37 & 32.62 & 20.33 & 47.36 & 0.92 & $\mathbf{1 0 1 . 2 3}$ & 0.00 & 43.57 & 55.58 \\
\hline \multirow{3}{*}{11} & $0-30$ & 7.84 & 4.35 & 14.38 & 9.01 & 19.35 & 0.54 & 43.28 & 0.00 & 18.40 & 24.19 \\
\hline & 30-50 & 7.85 & 4.07 & 12.35 & 8.55 & 18.47 & 0.39 & 39.76 & 0.00 & 17.85 & 21.43 \\
\hline & $50-150$ & 8.05 & 4.63 & 14.73 & 8.79 & 21.16 & 0.61 & 45.29 & 0.00 & 20.59 & 24.18 \\
\hline 12 & 0-30 & 7.16 & 14.74 & 51.45 & 24.31 & 67.63 & 1.23 & 144.62 & 0.00 & 61.60 & 80.67 \\
\hline \multirow{2}{*}{13} & $0-30$ & 7.35 & 6.28 & 21.36 & 12.29 & 27.17 & 0.56 & 61.38 & 0.00 & 26.02 & 34.17 \\
\hline & $30-60$ & 7.30 & 15.42 & 52.34 & 28.40 & 69.30 & 1.03 & 151.07 & 0.00 & 63.91 & 84.58 \\
\hline \multirow{2}{*}{14} & $0-25$ & 7.36 & 17.89 & 62.31 & 31.39 & 80.64 & 1.05 & 175.39 & 0.00 & 75.12 & 97.25 \\
\hline & $25-40$ & 7.30 & 20.81 & 66.35 & 37.04 & 92.76 & 2.09 & 198.24 & 0.00 & 86.13 & 108.46 \\
\hline \multirow{2}{*}{15} & $\mathbf{0 - 3 0}$ & 7.82 & 4.81 & 14.29 & 8.89 & 24.13 & 0.51 & 47.82 & 0.00 & 21.37 & 25.81 \\
\hline & $30-60$ & 7.75 & 5.47 & 17.28 & 10.86 & 25.31 & 0.63 & 54.08 & 0.00 & 23.87 & 29.74 \\
\hline & 0-30 & 8.35 & 6.73 & 20.18 & 14.63 & 30.82 & 0.64 & 66.27 & 0.00 & 29.98 & 34.32 \\
\hline 16 & $30-60$ & 8.23 & 6.87 & 20.37 & 15.27 & 30.64 & 0.56 & 66.84 & 0.00 & 30.06 & 35.24 \\
\hline & $60-90$ & 8.29 & 5.84 & 19.43 & 13.15 & 25.08 & 0.53 & 58.19 & 0.00 & 26.62 & 30.29 \\
\hline & $90-150$ & 7.98 & 5.93 & 18.29 & 13.14 & 27.14 & 0.59 & 59.16 & 0.00 & 26.32 & 31.56 \\
\hline & 0-30 & 7.94 & 7.01 & 22.04 & 14.28 & 31.57 & 0.78 & 68.67 & 0.00 & 30.80 & 37.09 \\
\hline 17 & $30-60$ & 8.14 & 5.33 & 18.32 & 10.17 & 23.85 & 0.48 & 52.82 & 0.00 & 23.75 & 27.46 \\
\hline & $60-110$ & 8.12 & 3.85 & 12.17 & 7.74 & 18.72 & 0.31 & 38.94 & 0.00 & 17.45 & 20.21 \\
\hline & 0-30 & 8.34 & 3.93 & 11.57 & 8.83 & 18.55 & 0.26 & 39.21 & 0.00 & 17.19 & 20.38 \\
\hline 18 & $30-60$ & 8.01 & 7.23 & 22.04 & 13.66 & 33.37 & 0.67 & 69.74 & 0.00 & 32.50 & 35.89 \\
\hline & $60-150$ & 7.97 & 10.54 & 34.65 & 20.45 & 45.35 & 0.89 & 101.38 & 0.00 & 46.91 & $\mathbf{5 3 . 5 2}$ \\
\hline
\end{tabular}

Fayoum J. Agric. Res. \& Dev., Vol.25, No.1, January, 2011 
It is worthy to mention that most of the soil chemical properties are more related to the nature of soil origin, relief, texture, land use period, management practices, cropping pattern, irrigation water suitability and system through the different periods of cultivation. In general, soil salinity tended to increase in the most of the studied soil profiles, except of some scattered areas such as those represented by soil profile Nos. 8 (alluvial moderately low terraces), 1, 3, 4 (low pediplain terraces and toe slopes) which represent extension areas.

On the other hand, the rest of the studied soils that developed on the physiographic units under investigation are suffering from salinity (ECe > 12 $\mathrm{dS} / \mathrm{m})$. The later conditions are more associated with inherited salts during chemical weathering (profile Nos. 6 and 14), miss-management practices such as the absence of adequate soil drainage system and continuous lateral seepage from the relatively high areas (soil profile No. 10). Also, the distinct pattern of soil moisture distribution throughout the irrigation with saline water may produce a pronounced effect on soil salinization (soil profile No. 9). The distribution patterns of soluble ions and soil sodicity (ESP) were more associated with the intensive of weathering and dominance of soluble $\mathrm{Na}+$ that stimulated more displacement of $\mathrm{Ca}$ and $\mathrm{Mg}$ by $\mathrm{Na}$ on soil colloidal complexes.

\section{Soil Taxonomy:}

Using the aforementioned analytical data as well as Classification System of USDA (1975) and Keys to Soil Taxonomy (USDA, 2010), the obtained data in Table (6) show that the studied soil profiles could be classified into three orders, i.e., Aridisols, Entisols and Vertisols as well as their followed sequence classification levels. Most of the representative soil profiles for pediplain landscape (profile Nos. 1, 3, 5, 6, 7, 11, 13, 14 and 15), in addition to some localities developed on alluvial terraces unit (soil profile No.10) could be classified into order of Aridisols, which has two sub-orders of Gypsids and Calcids, and two great groups of Calcigypsids and Haplocalcids; three sub-groups of Typic Calcigypsids Lithic Calcigypsids and Typic Haplocalcids as well as eight families, as follows:

a. Typic Calcigypsids loamy skeletal, mixed, hyperthermic (profile Nos. 1 and 7), sandy, mixed, (profile Nos. 3 and 11), sandy skeletal, mixed, hyperthermic (profile Nos. 5 and 13) and sandy mixed, hyperthermic (profile No.15).

b. Lithic Calcigypsids, sandy skeletal, mixed, hyperthermic (profile Nos. 6, and 12) and loamy skeletal, mixed, hyperthermic, (profile No.14) in the pediplain unit.

c) Typic Haplocalcids, clayey over sandy, mixed, hyperthermic of fluvio-lacutrine deposits (profile No.17) and Typic Haplocalcids, clayey, mixed, hyperthermic in the alluvial marches unit (profile No. 10).

Most of the alluvial plain soils and the sandy ones of pediplain are characterized by no evidence of development for pedogenic horizons and could be distinguished under Entisols. The Entisols order has two sub-orders of Orthents and Fluvents, two great groups of Torriorthents and Torrifluvents and two sub-groups of Typic Torriorthents and Typic Torrifluvents, as well as five families, as follows:

a. Sandy over clayey, mixed, hyperthermic (profile No. 2), Sandy skeletal, mixed, hyperthermic, moderately deep over limestone (profile No. 4) and sandy, mixed, hyperthermic (profile No. 18).

b. Fine loamy, mixed, hyperthermic (profile No. 9) and clayey, mixed, hyperthermic (profile No. 16).

Soils of the alluvial plain moderately low terraces (Pl 113) have the evidence of clayey soil and slickensides phenomenon that meet the requirements of Vertisols order (profile No. 8).

Fayoum J. Agric. Res. \& Dev., Vol.25, No.1, January, 2011 
Table (6): Soil taxonomic units of the studied soil profiles.

\begin{tabular}{|c|c|c|c|c|c|c|}
\hline 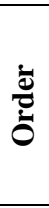 & 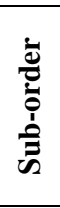 & 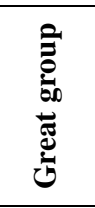 & 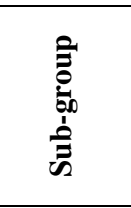 & 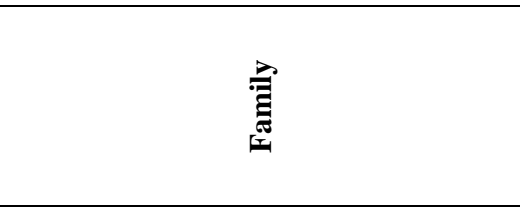 & 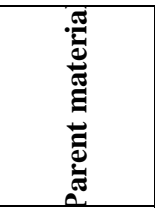 & $\frac{\mathscr{e}}{0}$ \\
\hline \multirow{8}{*}{$\frac{n}{8}$} & \multirow{6}{*}{$\frac{n}{\overbrace{0}^{2}}$} & \multirow{6}{*}{ 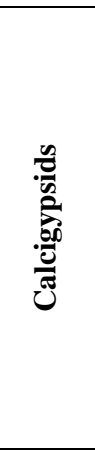 } & \multirow{4}{*}{ 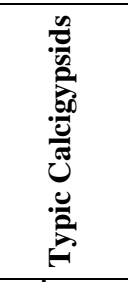 } & $\begin{array}{c}\text { Loamy skeletal, mixed, hyperthermic, } \\
\text { moderately deep over limestone }\end{array}$ & \multirow{6}{*}{$\begin{array}{l}\text { Eocene } \\
\text { limestone }\end{array}$} & 1 and 7 \\
\hline & & & & Sandy, mixed, hyperthermic & & 3 and 11 \\
\hline & & & & $\begin{array}{l}\text { Sandy skeletal, mixed, hyperthermic, } \\
\text { moderately deep over limestone }\end{array}$ & & 5 and 13 \\
\hline & & & & $\begin{array}{c}\text { Sandy, mixed, hyperthermic, } \\
\text { moderately deep over limestone }\end{array}$ & & 15 \\
\hline & & & $\frac{1}{\frac{1}{\pi}}$ & $\begin{array}{c}\text { Sandy skeletal, mixed, hyperthermic, } \\
\text { shallow over limestone }\end{array}$ & & 6 and 12 \\
\hline & & & 㞭 & Loamy skeletal, mixed, hyperthermic & & 14 \\
\hline & \multirow{2}{*}{$\frac{\mathscr{0}}{\tilde{e}}$} & \multirow{2}{*}{ 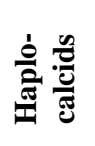 } & \multirow{2}{*}{ 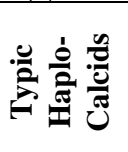 } & $\begin{array}{c}\text { Clayey over sandy, mixed, } \\
\text { hyperthermic }\end{array}$ & $\begin{array}{c}\text { Fluvio- } \\
\text { lacustrine }\end{array}$ & 17 \\
\hline & & & & Clayey, mixed, hyperthermic & Alluvium & 10 \\
\hline \multirow{5}{*}{ 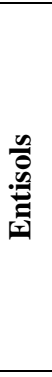 } & \multirow{3}{*}{ 苞 } & \multirow{3}{*}{ 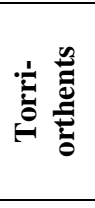 } & \multirow{3}{*}{ 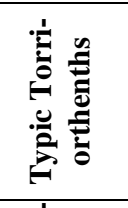 } & $\begin{array}{c}\text { Sandy over clayey, mixed, } \\
\text { hyperthermic }\end{array}$ & \multirow{3}{*}{$\begin{array}{c}\text { Fluvio- } \\
\text { lacustrine }\end{array}$} & 2 \\
\hline & & & & $\begin{array}{l}\text { Sandy skeletal, mixed, hyperthermic, } \\
\text { moderately deep over limestone }\end{array}$ & & 4 \\
\hline & & & & Sandy, mixed, hyperthermic, & & 18 \\
\hline & \multirow{2}{*}{ 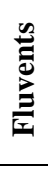 } & \multirow{2}{*}{ 党 } & \multirow{2}{*}{ 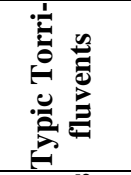 } & Fine loamy, mixed, hyperthermic, & \multirow[b]{3}{*}{ Alluvium } & 9 \\
\hline & & & & Clayey, mixed, hyperthermic & & 16 \\
\hline $\begin{array}{l}\frac{n}{0} \\
\stackrel{0}{0} \\
\substack{0 \\
0} \\
2\end{array}$ & $\stackrel{0}{0}$ & 完 & 둘 & Clayey, smectitic, hyperthermic & & 8 \\
\hline
\end{tabular}

\section{Land suitability for agricultural irrigated soils:}

\section{a. Current land suitability:}

The current suitability of the studied soils was estimated by matching between the present soil characteristics and their ratings as calculated by using the parametric system outlined by Sys and Verheye (1978), as shown in Table (7). Suitability indices and classification of the studied soils developed on the studied different physiographic units reveal that there are four soil suitablility classes, i.e., highly (S1), moderately (S2), marginally (S3) and not suitable (N1), besides nine subclasses, i.e., N1 $\mathrm{s}_{1} \mathrm{~s}_{2} \mathrm{~s}_{3} \mathrm{n}, \mathrm{S} 3 \mathrm{~s}_{1} \mathrm{~s}_{2} \mathrm{~s}_{4} \mathrm{n}, \mathrm{S} 3 \mathrm{~s}_{1} \mathrm{n}, \mathrm{S} 3 \mathrm{~s}_{1}, \mathrm{~S} 2 \mathrm{n}, \mathrm{S} 2 \mathrm{~s}_{1}, \mathrm{~S} 2 \mathrm{ws}_{1} \mathrm{~s}_{2}, \mathrm{~S} 2 \mathrm{~s}_{4} \mathrm{n}$ and $\mathrm{S} 1$ were recognized in the studied area. The soils of these sub-classes are suffering from some soil properties as soil limitations, i.e., soil texture $\left(\mathrm{s}_{1}\right)$, soil depth $\left(\mathrm{s}_{2}\right)$, $\mathrm{CaCO}_{3}$ content $\left(\mathrm{s}_{3}\right)$ and gypsum content $\left(\mathrm{s}_{4}\right)$ as well as salinity/alkalinity (n), with different intensity degrees. Also, it is evident that some localities of pediplain units (soil profile Nos. 1, 4 and 13) have a slight intensity of topography $(\approx 90)$. The obtained values of suitability indices show that some of the soils developed on the

Fayoum J. Agric. Res. \& Dev., Vol.25, No.1, January, 2011 
physiographic units of the alluvial high terraces (soil profile No. 16) could be evaluated as highly suitable (S1).

Table (7): Soil limitations and rating indices for the studied soil profiles.

\begin{tabular}{|c|c|c|c|c|c|c|c|c|c|c|c|}
\hline \multirow[b]{2}{*}{ 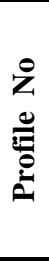 } & \multirow{2}{*}{ 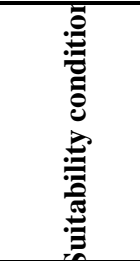 } & \multirow[b]{2}{*}{ 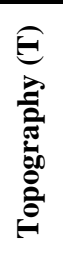 } & \multirow[b]{2}{*}{ 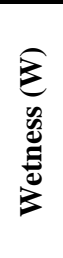 } & \multicolumn{4}{|c|}{ Physical characteristics } & \multirow{2}{*}{ 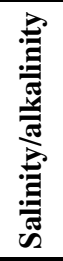 } & \multirow[b]{2}{*}{ 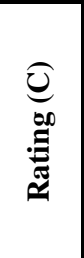 } & \multicolumn{2}{|c|}{ Land suitability } \\
\hline & & & & 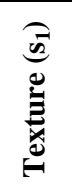 & 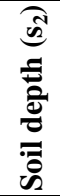 & 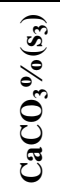 & 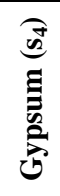 & & & Class & Subclass \\
\hline \multirow{2}{*}{1} & Current & 80 & 100 & 50 & 60 & 75 & 90 & 100 & 11.34 & $\mathbf{N} 2$ & $\mathbf{T}, \mathbf{s}_{1}, \mathbf{s}_{2}, \mathbf{s}_{3}, \mathbf{s}_{4}$ \\
\hline & Potential & 100 & 100 & 50 & 60 & 75 & 90 & 100 & 17.21 & $\mathbf{N 2}$ & $\mathbf{s}_{1}, \mathbf{s}_{2}, \mathbf{s}_{3}, \mathbf{s}_{\mathbf{4}}$ \\
\hline \multirow{2}{*}{2} & Current & 100 & 100 & 90 & 100 & 100 & 100 & 95 & 85.50 & S1 & -- \\
\hline & Potential & 100 & 100 & 90 & 100 & 100 & 100 & 100 & 90.00 & S1 & -- \\
\hline \multirow{2}{*}{3} & Current & 90 & 100 & 60 & 100 & 90 & 85 & 100 & 45.90 & S3 & $\mathbf{T}, \mathbf{s}_{1}, \mathbf{s}_{2}, \mathbf{s}_{3}, \mathbf{s}_{4}$ \\
\hline & Potential & 100 & 100 & 60 & 100 & 90 & 85 & 100 & 45.90 & S3 & $\mathbf{s}_{1}, \mathbf{s}_{2}, \mathrm{~s}_{3}, \mathrm{~s}_{\mathbf{4}}$ \\
\hline \multirow{2}{*}{4} & Current & 80 & 100 & 30 & 50 & 70 & 90 & 100 & 5.59 & N2 & $T, s_{1}, s_{2}, s_{3}, s_{4}$ \\
\hline & Potential & 100 & 100 & 30 & 50 & 70 & 90 & 90 & 2.74 & N2 & $\mathbf{s}_{1}, \mathbf{s}_{2}, \mathbf{s}_{3}, \mathrm{~s}_{4}$ \\
\hline \multirow{2}{*}{5} & Current & 90 & 100 & 40 & 35 & 55 & 55 & 60 & 2.28 & N2 & $T, s_{1}, s_{2}, s_{3}, s_{4}, n$ \\
\hline & Potential & 100 & 100 & 40 & 35 & 55 & 85 & 100 & 6.45 & N2 & $s_{1}, s_{2}, s_{3}, s_{4}$ \\
\hline \multirow{2}{*}{6} & Current & 100 & 100 & 30 & 25 & 65 & 90 & 75 & 3.29 & $\mathbf{N 2}$ & \\
\hline & Potential & 100 & 100 & 30 & 25 & 65 & 90 & 100 & 4.38 & $\mathbf{N 2}$ & $\mathbf{s}_{1}, \mathbf{s}_{2}, \mathbf{s}_{3}, \mathbf{s}_{4}$ \\
\hline \multirow{2}{*}{7} & & 100 & 100 & 40 & 35 & 60 & 80 & 90 & 3.63 & $\mathbf{N 2}$ & \\
\hline & Potential & 100 & 100 & 40 & 35 & 60 & 100 & 100 & 7.34 & N2 & $\mathbf{s}_{1}, \mathbf{s}_{2}, \mathbf{s}_{3}, \mathbf{s}_{4}$ \\
\hline \multirow{2}{*}{8} & Current & 90 & 100 & 90 & 100 & 80 & 100 & 90 & 58.32 & S2 & $\mathbf{s}_{1}, \mathbf{s}_{2}, \mathbf{s}_{3}, \mathbf{s}_{\mathbf{4}}, \mathbf{n}$ \\
\hline & Potential & 100 & 100 & 90 & 100 & 85 & 100 & 100 & 76.50 & S1 & -- \\
\hline \multirow{2}{*}{9} & Current & 100 & 100 & 90 & 90 & 80 & 100 & 90 & 58.32 & S2 & $\mathbf{s}_{1}, \mathbf{s}_{2}, \mathbf{s}_{3}, \mathbf{s}_{\mathbf{4}}, \mathbf{n}$ \\
\hline & Potential & 100 & 100 & 90 & 90 & 80 & 100 & 100 & 64.80 & S2 & $\mathbf{s}_{1}, \mathbf{s}_{2}, \mathbf{s}_{3}, \mathbf{s}_{4}$ \\
\hline \multirow{2}{*}{10} & Current & 90 & 100 & 100 & 100 & 75 & 100 & 85 & 57.38 & S3 & $T, s_{1}, s_{2}, s_{3}, s_{4}, n$ \\
\hline & Potential & 100 & 100 & 100 & 100 & 75 & 100 & 100 & 75.00 & S2 & $\mathbf{s}_{1}, \mathbf{s}_{2}, \mathbf{s}_{3}, \mathbf{s}_{\mathbf{4}}$ \\
\hline \multirow{2}{*}{11} & Current & 100 & 100 & 70 & 100 & 75 & 55 & 90 & 23.39 & N1 & $\mathbf{s}_{1}, \mathbf{s}_{2}, \mathbf{s}_{3}, \mathbf{s}_{\mathbf{4}}, \mathbf{n}$ \\
\hline & Potential & 100 & 100 & 70 & 100 & 75 & 80 & 100 & 37.80 & $\mathbf{S 3}$ & $\mathbf{s}_{1}, \mathbf{s}_{2}, \mathbf{s}_{3}, \mathbf{s}_{4}$ \\
\hline \multirow{2}{*}{12} & Current & 100 & 100 & 30 & 25 & 50 & 85 & 85 & 1.08 & N2 & $\mathbf{s}_{1}, \mathbf{s}_{2}, \mathbf{s}_{3}, \mathbf{s}_{\mathbf{4}}, \mathbf{n}$ \\
\hline & Potential & 100 & 100 & 30 & 25 & 50 & 100 & 100 & 3.75 & $\mathbf{N 2}$ & $\mathbf{s}_{1}, \mathbf{s}_{2}, \mathbf{s}_{3}, \mathbf{s}_{4}$ \\
\hline \multirow{2}{*}{13} & Current & 80 & 100 & 40 & 35 & 65 & 85 & 85 & 3.16 & N2 & $T, s_{1}, s_{2}, s_{3}, s_{4}, n$ \\
\hline & Potential & 100 & 100 & 40 & 35 & 65 & 100 & 100 & 5.46 & N2 & $\mathbf{s}_{1}, \mathbf{s}_{2}, \mathbf{s}_{3}, \mathbf{s}_{4}$ \\
\hline \multirow{2}{*}{14} & Current & 100 & 100 & 60 & 30 & 65 & 60 & 80 & 4.49 & $\mathbf{N 2}$ & $\mathbf{s}_{1}, \mathbf{s}_{2}, \mathbf{s}_{3}, \mathbf{s}_{\mathbf{4}}, \mathbf{n}$ \\
\hline & Potential & 100 & 100 & 60 & 30 & 65 & 80 & 100 & 7.49 & N2 & $s_{1}, s_{2}, s_{3}, s_{4}$ \\
\hline \multirow{2}{*}{15} & Current & 100 & 100 & 40 & 35 & 65 & 80 & 95 & 5.53 & $\mathbf{N 2}$ & $\mathbf{s}_{1}, \mathbf{s}_{2}, \mathbf{s}_{3}, \mathbf{s}_{4}, \mathbf{n}$ \\
\hline & Potential & 100 & 100 & 40 & 35 & 65 & 100 & 100 & 7.28 & $\mathbf{N 2}$ & $\mathbf{s}_{1}, \mathbf{s}_{2}, \mathbf{s}_{3}, \mathbf{s}_{4}$ \\
\hline \multirow{2}{*}{16} & Current & 100 & 100 & 100 & 100 & 100 & 100 & 90 & 90 & S1 & -- \\
\hline & Potential & 100 & 100 & 100 & 100 & 100 & 100 & 100 & 100 & S1 & -- \\
\hline 17 & Current & 90 & 100 & 100 & 90 & 90 & 100 & 90 & 59.05 & S2 & $\mathbf{T}, \mathbf{s}_{2}, \mathbf{s}_{3}, \mathbf{s}_{\mathbf{4}}, \mathbf{n}$ \\
\hline 17 & Potential & 100 & 100 & 100 & 90 & 90 & 100 & 100 & 81.00 & S1 & -- \\
\hline & Current & 100 & 100 & 100 & 100 & 95 & 100 & 90 & 85.50 & S1 & -- \\
\hline 10 & Potential & 100 & 100 & 100 & 100 & 95 & 100 & 100 & 95.00 & S1 & -- \\
\hline
\end{tabular}

The alluvial plain and moderately high terraces, i.e., Pl 112, (soil profile No. 18 ) and pediplain low terraces ( $p$ p 213) could be evaluated as moderately suitable

Fayoum J. Agric. Res. \& Dev., Vol.25, No.1, January, 2011 
(S2), with slight soil limitations. On the other hand, some soils of alluvial plain terraces (profile Nos. 8 and 9), toe slopes and foot slopes of pediplain (profile Nos. 3 and 11) are evaluated as marginally suitable for irrigated agriculture. In addition, the index value indicates a currently not suitable class for some soils of marches Pl 116 (soil profile No. 10) and some soils of pediplain elongated hills and slopes piedmont unit (soil profile Nos. 6, 5 and 17), however, the representative soils have slight intensity of topography, severe intensity of soil texture and effective soil depth as well as moderate intensity for both $\mathrm{CaCO}_{3}$ content and soil salinity/alkalinity limitations.

\section{b. Potential land suitability:}

Land improvements are activities, which cause beneficial changes in the qualities of the land itself. They are classified as major land improvements. Further land improvements are required to correct or to reduce the severity of limitations exiting in the studied area, such as: a) Leveling of undulating surfaces, b) Leaching of soil salinity and reclamation of alkalinity existing in the soils, c) Construction of efficient open drainage ditches to enhance leaching process of the excess soluble salts as well as to accelerate the chemical remediation of soil sodicity, d) Continuous application of organic manure to improve soil physico-chemical properties and fertility status, e) Application of modern irrigation systems, i.e., drip and sprinkler to save a pronounced amount of irrigation water as well as to rise the irrigation efficiency.

It is applicable after executing specified major land improvements as proposed in the aforementioned agro-management practices according to their necessity. For establishing potential land suitability classification, the main land improvements for the studied area are considered for the land qualities of drainage, salinity and sodicity. The minor limitations can be improved under specific land management, concerning each of them. So, the obtained potential land suitability sub-classes were sorted for the maximum productive levels (supreme potential land suitability). These levels were designed to be guide charts and maps for the best land utilization alternatives, giving a possible maximum output. The potential land suitability data are shown in Table (7).

These adaptations can be described by applying the previous improvement practices, and in turn potential suitability of the studied soils indicates the existing of two suitability classes, i.e., highly (S1) and moderately (S2), however, soil suitable sub-classes of both alluvial flood plain terraces (soil profile Nos. 8,16 and 18) and pediplain foot slope and depression bottom (soil profile Nos. 2 and 11) improved from moderately suitable (S2) to highly suitable (S1).Soils of profiles Nos. 9 and 3 could be improved from marginally suitable (S3) to moderately suitable (S2). The later subclass represents some soil profiles developed on some of the different studied physiographic units with severe to moderate intensity of soil texture (relatively coarse) as soil limitations. The severity of soil texture (sand) can be corrected in these subclasses by application of organic and inorganic soil amendments as well as drip irrigation system to sustain soil moisture content at favourable conditions for grown plants.

\section{CONCLUSION:}

This work was carried out in order to study the possibility of using the Spectral Angler Mapper technique to classify different land covers in the studied area and to identify major changes that have taken place during 21 years (the period of the study). The study area was classified into four classes and a confusion matrix

Fayoum J. Agric. Res. \& Dev., Vol.25, No.1, January, 2011 
based on ground truth data used to verify the thematic maps. A post classification comparison approach was used to detect land use and cover changes.

The results showed considerable potential of the Spectral Angler Mapper technique in classifying land use and cover. The main land cover changes in the study area could be summarized thus: agricultural areas, water and settlement increased considerably with a high rate of annual increase during the entire study period; there is a substantial decrease in the other land use and land cover. Such base information can help decision-makers and land planners in the process of determining priority areas for the implementation of management plans or soil reclamation measures at regional level to avoid adverse environmental effects. Moreover, the data of the current study were created to update and support the local knowledge, particularly the best use of land whether be under demand for agriculture use or be planned for later on use. It means that the obtained findings represent the best adaptation between certain land units with specific soil properties to give the maximum outputs. Also, identifying the physiographic features of an unique area in the north-western desert outskirt of El-Fayoum depression by mapping them to be a digital model in a harmony of physiographic and soil data set, serving the extrapolation approach when other areas will be under study.

\section{REFERENCES:}

Congalton R.G. (1991). A review of assessing the accuracy of classifications of remotely sensed data. Remote Sensing of Environment, 37: 35-46.

Coppin P.; I. Jonckheere; K. Nackaerts; B. Muys and E. Lambin (2004). Digital change detection methods in ecosystem monitoring: a review, International Journal of Remote Sensing25 (9) (2004), pp. 1565-1596

Dai, X.L. and S. Khorram (1999). Remotely sensed change detection based on artificial neural networks. Photogramm. Eng. Rem. Sen.65, pp. 1187-1194.

Gee, G.W. and J.W. Bauder (1986). Particle size analysis. In: Methods of Soils Analysis, Part I, Klute, A. (Ed.), Agronomy No. 9.

Kraus N.C. (1999). Analytical model of spit evolution at inlets, Proceedings of Coastal Sediments'99, ASCE (1999), pp. 1739-1754.

Klute, A. (1986). Methods of Soil Analysis, Part I., 2nd Edition , American Society of Agronomy, Madison, Wisconsin, USA.

Liu D., P. Mausel; E. Brondízio and E. Moran (2002). Assessment of atmospheric correction methods for Landsat TM data applicable to Amazon basin LBA research, International Journal of Remote Sensing23 (13) (2002), pp. 2651-2671.

Liu D. (2004). P. Mausel, E. Brondizio and E. Moran (2004). Change detection techniques, International Journal of Remote Sensing 25 (12), pp. 2365-2407.

Macleod, R.D. and R.G. Congalton (1998). Quantitative comparison of change detection algorithms for monitoring eelgrass from remotely sensed data.Photogrammetric Engineering and Remote Sensing 64, pp. 207-216.

Mas, J.F. (1999). Monitoring land-cover changes : a comparison of change detection techniques. International Journal of Remote Sensing , 20 (1): 139152.

Munsell Soil Colour Charts (1975). Munsell Colour, Macbeth Division of Kollmorgen Corp. 2441, North Calvert Street, Baltimore, Maryland, USA.

Page, A.I.; Miller R.H. and D.R. Keeney (1982). Methods of Soil Analysis : Part -2 Chemical and Microbiological Properties . 2nd Ed. Amer. Soc. Agron. Madison, Wisconsin, USA

Fayoum J. Agric. Res. \& Dev., Vol.25, No.1, January, 2011 
Ragab, M.A. (2001). The effect of calcium carbonate content and size distribution on soil properties, drainage efficiency and crop yield under different designs of tile drainage system. Ph. D. Thesis. Fac. of Agric., Ain Shams Univ., Egypt.

Said, R. (1990). The Geology of Egypt. Published for the Egyptian Central Petroleum Corporation, Conoco Hurghada Inc. by Balkema, A.A., Roterdam, The Netherlands.

Singh A. (1989). Digital change detection techniques using remotely-sensed data, International Journal of Remote Sensing10 (6) (1989), pp. 989-1003.

Sys, C. and W. Verheye (1978). An attempt to the evaluation of physical land characteristics for irrigation according to the FAO Framework for land Evaluation. Int. Train. Cent. for Post Graduate, Soil Scientist

U.S.D.A. (1975). Soil Taxonomy Basic System of Soil Classification. U.S. Dept., Agric. Handbook No. 436, USDA. Washington, D.C. 20402.

USDA (2003). Soil Survey Manual. United State, Department of Agriculture, Handbook 18, U.S. Gov. Print Off., Washington, DC., USA

U.S.D.A. (2010). Keys to Soil Taxonomy. 11th (Ed), United States Department of Agriculture, USA.

Weismiller, R.A.; S.J. Kristof; D.K. Scholz; P.E. Anuta and S.A. Momen (1977). Change detection in coastal zone environments, Photogrammetric Engineering and Remote Sensing43 (1977), pp. 1533-1539.

Yuhas, R.H.; A.F.H. Goetz and J.W. Boardman (1992). Discrimination among semiarid landscape endmembers using the Spectral Angle Mapper (SAM) algorithm. Summaries of the Third Annual JPL Airborne Geoscience Workshop, 1 June, Pasadena, CA, Jet Propulsion Laboratory, 147-149.

Zhou, L., R.E. Dickinson; Y. Tian; X. Zeng; Z.L. Yang and M. Shaikh (2003). Comparison of seasonal and spatial variations of albedos from Moderate-Resolution Image Spectroradiometer (MODIS) and Common Land Model. J. Geophys, Res. 108 (D15): 4481.

Zinck, J.A. (1989). Physiography and soils., Lecture notes, ITC, Enschede, The Netherlands.

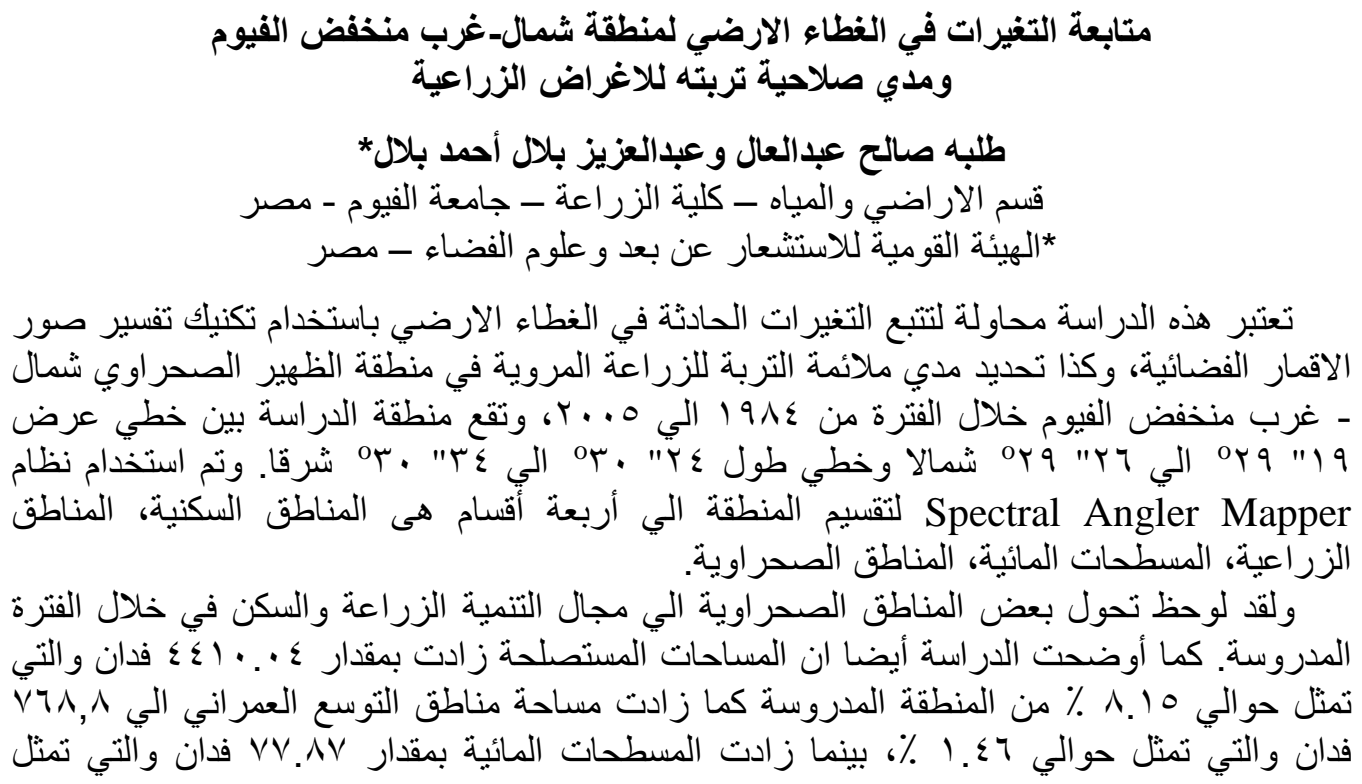

Fayoum J. Agric. Res. \& Dev., Vol.25, No.1, January, 2011 
Tolba S. Abdel Aal \& Abdel Aziz B.A. Belal

حو الي بr. • ٪؛ هذه الزيادة ربما تعزي الي التغيرات الجيوفيزيائية و الاجتماعية مثل الملوحة و القلوية

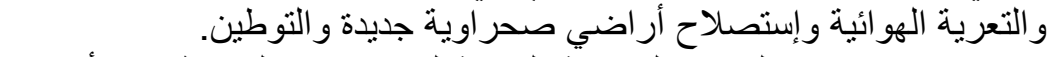

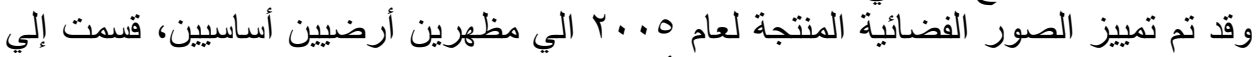
rا وحدة باستخدام تكنيك التفسير المرئي لصورة الأقمار الفضائية:

ETM7 (Enhanced Thematic Mapper 7) and applying the Landscape feature approaches.

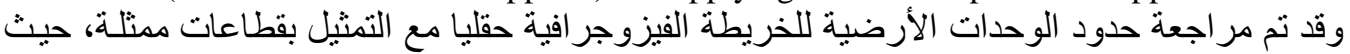

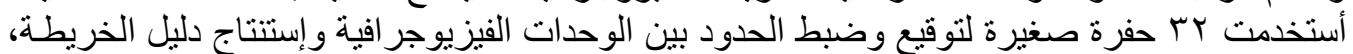

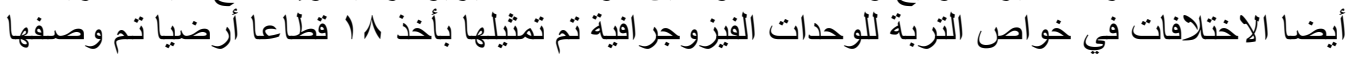

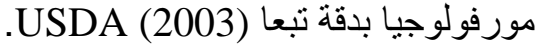
وقد تم تقسيم التربة تبعا (USDA (1975 and 2010) حيث وجد أن أراضيها تنتمى إلى ثلاث رتب، ست تحت مجمو عة كما يلي:

Aridisols (Typic Calcigypsids, Lithic Calcigypsids, and Typic Haplocalcids), Entisols (Typic Torriorthents and Typic Torrifluvents ) and Vertisols (Typic Haplotorrierts).

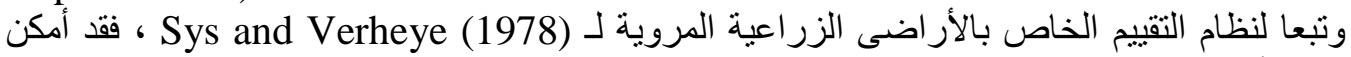

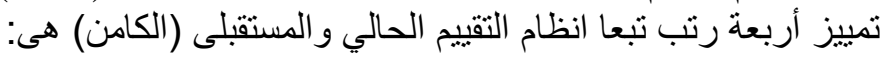
Highly (S1), moderately (S2), marginally (S3) and not suitable (N1 \& N2). 